\title{
Comparison Metrics for Time-histories: Application to Bridge Aerodynamics
}

\author{
Igor Kavrakov $^{1, *}$, Ahsan Kareem² ${ }^{2}$ and Guido Morgenthal ${ }^{3}$ \\ ${ }^{1}$ Research Associate, Chair of Modelling and Simulation of Structures, Bauhaus University Weimar, \\ Marienstr. 13, Weimar 99423, Germany. *Corresponding author, E-mail: igor.kavrakov@ uni-weimar.de \\ ${ }^{2}$ Robert M. Morgan Professor, NatHaz Modeling Labratory, University of Notre Dame, 156 Fitzpatrick \\ Hall, Notre Dame, IN 46556, USA. \\ ${ }^{3}$ University Professor, Chair of Modelling and Simulation of Structures, Bauhaus University Weimar, \\ Marienstr. 13, Weimar 99423, Germany.
}

\begin{abstract}
Wind effects can be critical for the design of lifelines such as long-span bridges. The existence of a significant number of aerodynamic force models, used to assess the performance of bridges, poses an important question regarding their comparison and validation. This study utilizes a unified set of metrics for a quantitative comparison of time-histories in bridge aerodynamics with a host of characteristics. Accordingly, nine comparison metrics are included to quantify the discrepancies in local and global signal features such as phase, time-varying frequency and magnitude content, probability density, nonstationarity and nonlinearity. Among these, seven metrics available in the literature are introduced after recasting them for time-histories associated with bridge aerodynamics. Two additional metrics are established to overcome the shortcomings of the existing metrics. The performance of the comparison metrics is first assessed using generic signals with prescribed signal features. Subsequently, the metrics are applied to a practical example from bridge aerodynamics to quantify the discrepancies in the aerodynamic forces and response based on numerical and semi-analytical aerodynamic models. In this context, it is demonstrated how a discussion based on the set of comparison metrics presented here can aid a model evaluation by offering deeper insight. The outcome of the study is intended to provide a framework for quantitative comparison and validation of aerodynamic models based on the underlying physics of fluid-structure interaction. Immediate further applications are expected for the comparison of time-histories that are simulated by data-driven approaches.
\end{abstract}

Final draft: Kavrakov, I., Kareem, A., and Morgenthal, G. (2020). Comparison Metrics for Time-Histories: Application to Bridge Aerodynamics. Journal of Engineering Mechanics, 146 (9), 04020093.

https://doi.org/10.1061/(ASCE)EM.1943-7889.0001811 


\section{INTRODUCTION}

With increasing spans of cable-supported bridges, wind-induced vibrations become the most prominent action on such structures. A significant number of aerodynamic models have been developed to describe the wind loads acting on a bridge deck for buffeting and flutter analyses. Traditionally, these models are semi-analytical (cf. e.g. Scanlan (1978), Chen and Kareem (2002), Chen and Kareem (2003), Chen and Kareem (2006), Chen and Kareem (2001), Diana et al. (2010), Wu and Kareem (2013c)). In the last two decades, models based on Computational Fluid Dynamics (CFD) also have received a considerable attention (cf. e.g. Ge and Xiang (2008), Morgenthal and McRobie (2002), Morgenthal et al. (2014, Kavrakov and Morgenthal (2018a)).

Having an abundant number of aerodynamic force models available, a logical question surfaces regarding their quality and ability to replicate aerodynamic phenomena. In the case of the semi-analytical models, this is directly related to their model assumptions, such as linearity or their disregard of fluid memory. On the other hand, the choice of discretization scheme and turbulence models plays a vital role in the quality of CFD simulations. Hence, several assessment studies have emerged that compare aerodynamic models or validating them with experimental data (cf. e.g. Petrini et al. (2007), Wu and Kareem (2013b), Kavrakov and Morgenthal (2017), Kavrakov and Morgenthal (2018b), Øiseth et al. (2011), Diana et al. (2013)). Moreover, the task group "Super-long span bridge aerodynamics" of the International Association of Bridge and Structural Engineering (IABSE) is currently establishing a benchmark example for computer code verification and model validation for buffeting and flutter analyses of bridges (Diana et al. 2019a; Diana et al. $2019 b)$. Within this group, results from different computer codes of various research groups and companies are compared within the activities of this IABSE working group.

Most of the aforementioned studies use the aerodynamic force or aeroelastic displacement as a basis for comparison. For time-domain analyses, this effectively constitutes a comparison of two time-histories. Thus, a comparison is typically conducted based on the root mean square (RMS) or the peak, and by looking at time-histories or power spectral densities. However, these quantities are insufficient to compare the discrepancies of the embedded nonlinear features in the output of advanced aerodynamic models (cf. e.g. Carassale et al. (2014), Wu and Kareem (2011), Wu and Kareem (2013c), Diana et al. (2010)). Therefore, each comparative study is carried out case-by-case and a unified framework for comparison of time-histories in bridge aerodynamics is not available.

The literature on methods for comparing time-histories is vast. Without being exhaustive, former studies can be found in field of mechanical systems (Jiang and Mahadevan 2010), cavitation in fluid-structure interaction (Sprague and Geers 2004), seismology (Kristeková et al. 2009), geophysics (Willmott et al. 1985), economics (Bliemel 1973), shock of floating platforms (Teferra et al. 2014), crash simulations in vehicle designs (Sarin et al. 2010), etc. For data-driven simulations of downburst winds, Wang et al. (2013) have utilized a number of schemes for validation, including metrics like Rényi entropy and fractal dimension. The commonality of all methods is that the similarity of the time-history is measured by an absolute or relative metric. These metrics are used to quantify the discrepancies in certain signal features, such as magnitude, phase, frequency content, probability distribution function (PDF), etc. A feature is a distinctive or characteristic measurement, transform, structural component extracted from a segment of a pattern (Cvetkovic et al. 2008). Hora and Campos (2015) give a relatively comprehensive review of available metrics. Generally, the selection of metrics for comparison is based on the field of application as different embedded signal features are of interest.

In light of the previous overview, this study utilizes a set of comparison metrics for multi-criteria model assessment in bridge aerodynamics and aeroelasticity. The metrics are tailored to quantify global and local discrepancies of two time-histories, which are of interest for aeroelastic/aerodynamic analyses. A total of nine metrics are constructed on a uniform basis. Seven of these metrics are adapted from former studies and recast to meet the needs of the current application, including peak, RMS, phase, magnitude, PDF, wavelet and frequency-normalized wavelet metrics. Moreover, two additional metrics are introduced to quantify the 
discrepancies in possible nonstationary and quadratic nonlinearity of the signals. These two metrics build upon on existing methods for testing stationarity with wavelet surrogates and nonlinearity with the wavelet bispectrum, respectively.

By constructing this set of comparison metrics, this study aims to address the question: How to meaningfully compare two force/response time-histories in bridge aerodynamics? In this regard, the entire set of comparison metrics is intended to represent a unified and standard way to quantitatively compare not only the typical signal features such as peak and RMS but also the nonlinear and nonstationary signal features. All metrics are discussed from the perspective of their relation to the underlying physical processes that take place in the wind-bridge interaction.

The study is organized as follows: Initially, peculiar signal features in bridge aerodynamics are discussed. Then, the comparison metrics are introduced. This is followed by examination of their performance via controlled numerical experiments using generic signals. Finally, the metrics are applied to an example from bridge aerodynamics in terms of dynamic response and force time-histories. In closing, a brief summary and conclusions are given.

\section{EMBEDDED SIGNAL FEATURES IN BRIDGE AERODYNAMICS}

Looking at the wind-bridge interaction as an input/output system, it is characterized by properties such as memory, nonlinearity and stochastic features. These properties are manifested in peculiar signal features embedded in the output.

The system memory (fluid memory) affects the output aerodynamic forces by introducing a time lag and amplitude scaling of the fluctuating pressure coefficients due to input motion or gusts. As a result, the aerodynamic forces experience a hysteretic behavior with frequency-dependent shape for linear models (cf. Fig. 1). According to the flat plate unsteady theory (cf. e.g. Fung (1993)), this is a consequence of the uneven distribution of the bound circulation. For nonlinear aerodynamic systems, the fluid memory effect can also be time-dependent, i.e., fading fluid memory (cf. e.g. Carassale et al. (2014), Wu and Kareem (2011)). Intuitively, fading memory means that current output depends more on the recent input than remote past (Shamma and Zhao 1993). This effectively challenges the infinite wake assumption in linear aerodynamics and influences the force phase and amplitude.

Apart from the fading fluid memory, nonlinear effects can be manifested through amplitude-dependence, nonGaussianity, and nonstationarity of the aerodynamic forces/response. As a result of the nonlinear inputoutput relationship for large angles of attack, the aerodynamic forces may experience sub-and superharmonics for a single-frequency input (cf. Fig. 1) (Li and Kareem 1990; Huang et al. 2014; Diana et al. 2010). Further,
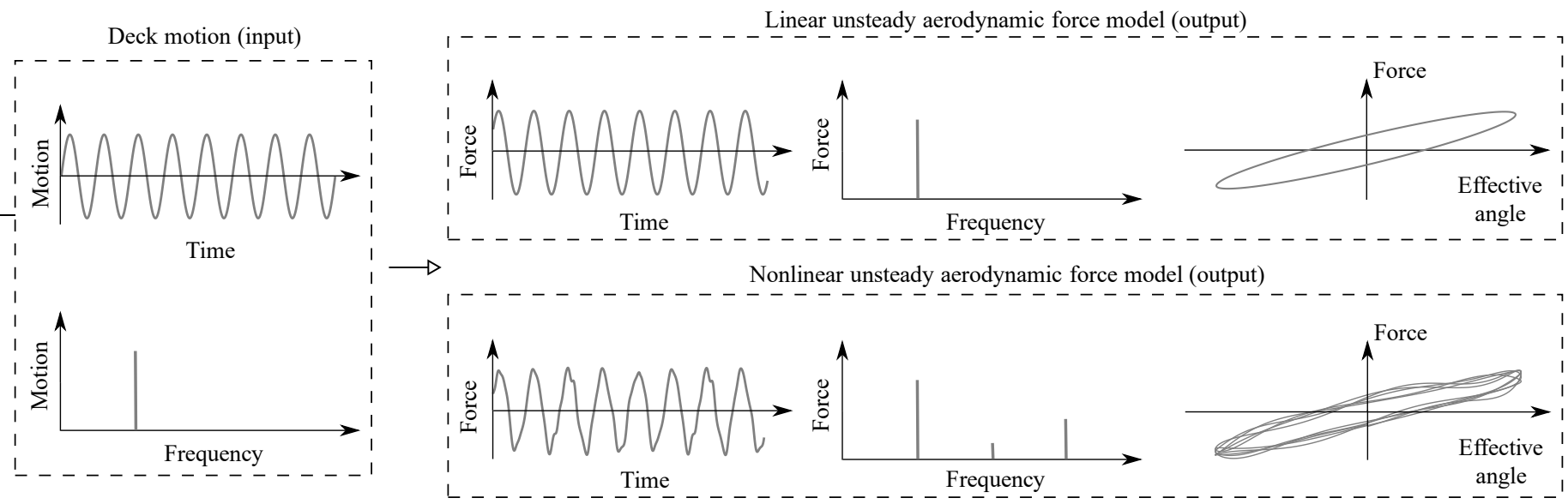

Fig. 1. Linear and nonlinear aerodynamic forces acting on a bridge deck due to a sinusoidal input motion. 
the input-output amplitude ratio at the forcing frequency may vary depending on the input amplitude. This is manifested through the amplitude dependence of the aerodynamic coefficients.

In regions with massive flow separation, the PDF of the local pressure can differ significantly from a Gaussian despite a Gaussian free-stream turbulence (Kareem and $\mathrm{Wu}$ 2013). This yields force peaks that cannot be directly related to the RMS and large higher-order moments of the output PDF (e.g. skewness and kurtosis). Such nonGaussian features can be observed in the local pressure variation on rooftop cladding (Gurley and Kareem 1997) or the tail of the PDF of the buffeting forces acting on a bridge deck (cf. Fig. 2).

Despite having a stationary input, a stable nonlinear aerodynamic system can result in a nonstationary output in terms of amplitude and frequency. In bluff-body aerodynamics, this is related to the nonlinearity of the aerodynamic forces due to strong stationary wind gusts and large oscillations (Kavrakov and Morgenthal 2018b). Large angles of attack often lead to a formulation of a leading-edge vortex that entrains vorticity as it is convected along the deck (cf. Fig. 3), which effectively induces high-pressure peaks. Moreover, nonstationary amplitude modulations in the response are also present during flutter, or even in terms of frequency modulation in the post-flutter limit cycle oscillations (cf. e.g. Abbas et al. (2017), Kavrakov and Morgenthal (2018b)).

Additional broadband high-frequency content in the forces can appear as a consequence of vortex shedding and/or interior noise. The latter entails chaotic (stochastic) phenomena such as wake instability, laminar-turbulence transition, local separation, and reattachment (Wu and Kareem 2015; Kavrakov and Morgenthal 2018b). These local turbulence effects can also lead to a nonstationary hysteresis behavior for a sinusoidal input motion (cf. Fig. 1) (Zhang et al. 2017).

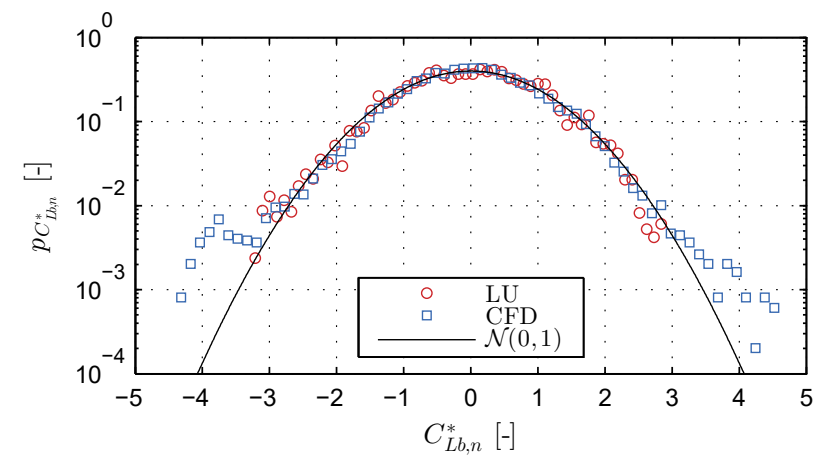

Fig. 2. Histogram (approximate PDF) of the normalized (standard score) buffeting lift coefficient $C_{L b, n}^{*}$ for a linear semi-analytical model (LU) and a CFD (nonlinear) model. The input is a Gaussian free-stream turbulence and the deck is static. For comparison, a Gaussian distribution with zero mean and unit variance $\mathcal{N}(0,1)$ is included. (Data from Kavrakov and Morgenthal 2018b)

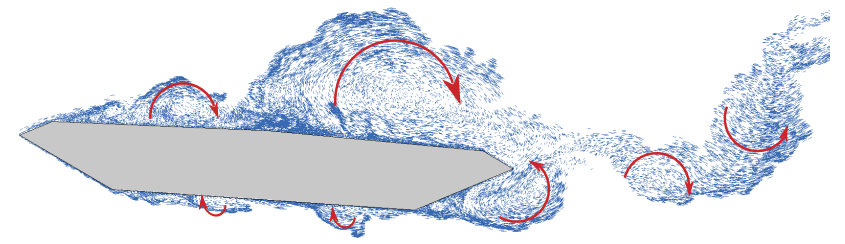

Fig. 3. Instantaneous particle map for deck oscillations at high angle of attack, resulting in leading edge vortex separation. (Data from Kavrakov and Morgenthal 2018b). 


\section{COMPARISON METRICS}

The general idea of a comparison metric between two time-histories is to quantify the discrepancies of a particular feature between two signals. Considering two time-histories, $x(t)$ and $y(t)$ with duration $T$, a comparison metric is denoted as $M^{x, y}=M(x, y)$, where $x(t)$ is the reference signal. Since various features of the signals (e.g. phase, magnitude) can attain magnitudes of a different order, it is beneficial to construct the comparison metrics so that their values are in the interval between 0 and 1 . In such way, a comparison metric amounting to $M^{x, y}=1$ indicates that there are no discrepancies in a particular feature of the timehistory, while $M^{x, y}=0$ indicates that the difference is infinite. To facilitate this, the comparison metrics are constructed in a unified manner using an exponential function with a negative exponent as follows:

$$
M^{x, y}=M(x, y)=e^{-\lambda A(x, y)},
$$

where $A(x, y)$ is a relative exponent and $\lambda$ is a sensitivity parameter. The relative exponent $A(x, y)$ is defined in such manner to account for the discrepancies of a particular feature of the time-histories, while the sensitivity parameter $\lambda \geq 0$ is introduced to adjust the sensitivity of different metrics. A total of nine metrics are considered for this study with a focus on the underlying physics of wind-bridge interaction. The following metrics are included: (i) phase $M_{\phi}$, (ii) peak $M_{p}$, (iii) RMS $M_{\mathrm{rms}}$, (iv) magnitude $M_{m}$, (v) PDF $M_{\mathrm{pdf}}$, (vi) wavelet $M_{w}$, (vii) frequency-normalized wavelet $M_{w f}$, (viii) stationarity $M_{s}$, and (ix) wavelet bispectrum $M_{b}$ metrics. In what follows, all of the metrics are be defined by their relative exponent.

\section{Phase, peak, and root mean square metrics}

First, metrics based on global and averaged signal features are investigated, including the phase, peak and RMS metrics. The phase metric can be considered as an indicator of the extent of the ability of an aerodynamic model to capture the fluid memory effects. Herein, the phase metric introduced by Sarin et al. (2010) is used since its definition using an exponential function is compliant with the unified manner of construction of the comparison metrics in Eq. (1). The relative exponent for this metric yields the following:

$$
A_{\phi}=\frac{t_{\mathrm{lag}}}{T_{c}}
$$

where mean time delay $t_{\text {lag }}$ is obtained as follows:

$$
t_{\mathrm{lag}}=\arg \max [x(t) \star y(t)],
$$

where the star " $\star$ " denotes cross-correlation. The normalization time $T_{c}$ is a time that is considered to be a significant delay between the signals; hence, it is case-dependent, as discussed later. This metric accounts for the mean phase discrepancy between two signals. For frequency-modulated signals, the phase metric might not carry an effective meaning.

The peak values of the aeroelastic response are the design criteria for bridges. Thus, they are of major importance for aerodynamic time-histories, particularly in cases with high aerodynamic nonlinearities that influence the peak factor.

The relative exponent for the peak metric is defined as follows:

$$
A_{P}=\frac{|\max | x(t)|-\max | y(t)||}{\max |x(t)|} .
$$

The utility of the RMS for time-histories is due to its physical interpretation as it is closely related to the signal energy. The RMS has shown to be a useful signal feature for linear aerodynamic models, particularly when comparing between the frequency- and time-domain for a Gaussian free-stream turbulence Øiseth et al. (2011). 
The relative exponent for the RMS metric is obtained as the relative difference of the RMS of the signals, which yields the following:

$$
A_{\mathrm{rms}}=\frac{\left|\sqrt{\frac{1}{T} \int_{0}^{T}[x(t)]^{2} \mathrm{~d} t}-\sqrt{\frac{1}{T} \int_{0}^{T}[y(t)]^{2} \mathrm{~d} t}\right|}{\sqrt{\frac{1}{T} \int_{0}^{T}[x(t)]^{2} \mathrm{~d} t}} .
$$

\section{Magnitude metric}

The peak and RMS metrics are based on a global and averaged amplitude discrepancies, respectively. To further study the differences of the magnitude in a time-localized manner, a relative magnitude metric is needed. A straightforward manner to determine the local amplitude discrepancies would be to compute the RMS deviation of the signals. However, a simple subtraction of two signals might result in large values if the signals are phase-shifted or in the presence of interior noise due to local effects and vortex shedding, which would unrealistically exaggerate the situation.

Sarin et al. (2010) propose to preprocess the original time-histories utilizing the dynamic time warping (DTW) algorithm to alleviate some of the local phase-shifts and very high frequencies. Utilizing the DTW, the peaks of the signals are aligned in a nonlinear fashion by stretching, but not scaling. Taking this into account, the relative exponent for the magnitude metric yields the following:

$$
A_{m}=\frac{\sqrt{\frac{1}{N_{w}} \sum_{j=1}^{N_{w}}\left(x_{w, j}-y_{w, j}\right)^{2}}}{\sqrt{\frac{1}{N_{w}} \sum_{j=1}^{N_{w}}\left(x_{w, j}\right)^{2}}},
$$

where $x_{w, j}$ and $y_{w, j}$ for $j=\left\{1, \ldots, N_{w}\right\}$ are the warped versions of the original discretized time-histories $x_{m}$ and $y_{m}$ for $m=\{1, \ldots, N\}$, respectively, and $N_{w}$ is the number of warped steps, which is not necessarily the same with the number of time-steps $N$ of the original discretized signals.

To illustrate the effect of DTW, consider two biharmonic signals with distinct phase-shift for each harmonic and additional Gaussian noise (cf. Fig. 4, left). The DTW algorithm (cf. e.g. Müller (2007)) consists of initially computing an accumulated cost matrix $C_{m n}=\min \left(C_{m-1, n-1}, C_{m, n-1}, C_{m-1, n}\right)+d\left(x_{m}, y_{n}\right)$ for $m, n=\{1, \ldots, N\}$, based on a local distance measure $d\left(x_{m}, y_{n}\right)$ such as, e.g., Euclidean distance. A sequence of pairs of indices $w_{j}=[m, n]$ is sought which minimizes a cumulative $\operatorname{cost} C_{p}=\sum_{1}^{N_{w}} C\left[w_{j}\right]$. The sequence $w_{j}$ is the optimal warping path, which is subjected to the following constraints: (i) $w_{j}$ is
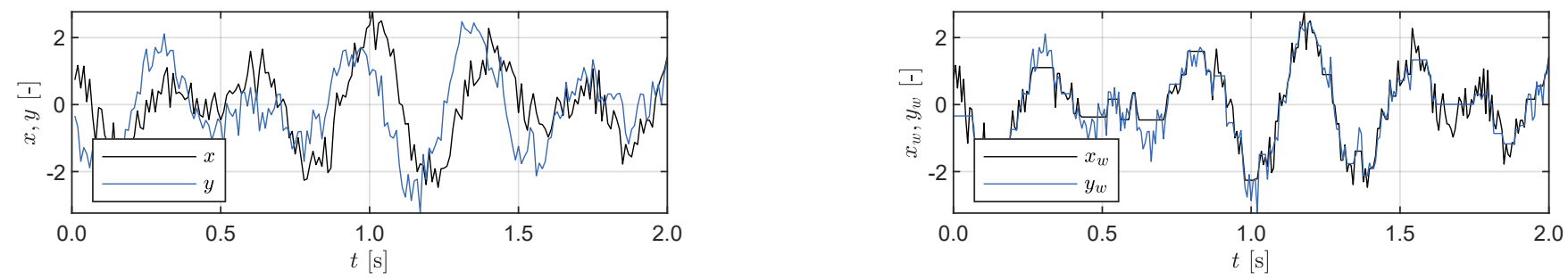

Fig. 4. Original (left) and warped (right) time-histories. 


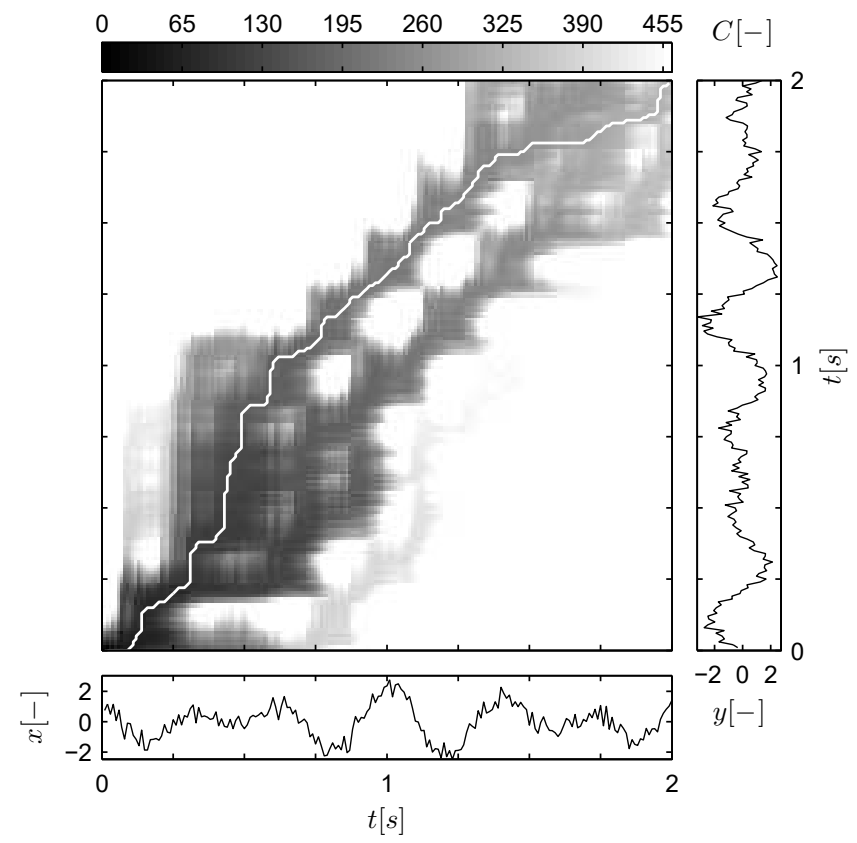

Fig. 5. Accumulated cost matrix $C$ of the DTW algorithm. The white line represents the optimal warping path.

monotonically increasing, (ii) $w_{j}$ progresses one step at a time, (iii) the boundary values are $w_{1}=[1,1]$ and $w_{N_{w}}=[N, N]$. The lower the cumulative cost, the more the signals are similar to each other, and visa-versa. For the particular example, the accumulated cost matrix and the optimal warping path (the white line) are shown in Fig. 5. Based on the ordered pair of indices in $w_{j}$, the values of the discrete signals $x_{m}$ and $y_{m}$ are correspondingly ordered, resulting in the warped signals in Fig. 4 (right). The warped signals can be directly compared with respect to the isolated magnitude discrepancies. The cumulative cost is an indicator of the amount of shifting of the signals to obtain their warped counterparts. However, this is not taken into account within the magnitude metric as it is assumed that $C_{p}$ corresponds to the phase and local frequency shifts, which are not of interest for this metric.

\section{Probability density function metric}

The variation between the input and output PDF is a property of nonlinear models; hence, it represents an important signal feature to quantify the nonGaussian properties in bridge aerodynamics and aeroelasticity.

In statistics, two PDFs are compared by utilizing statistical divergence. There are a number of statistical divergences which quantify the discrepancy between the PDFs, such as the Kullback-Leiber divergence, Jensen-Shannon divergence, Hellinger distance, etc. (cf. e.g. (Pardo 2006)). Herein, the Bhattacharyya distance $D_{B}$ is utilized to formulate the PDF metric as it is commonly used to measure the overlapping degree of PDF (cf e.g. (Nielsen and Boltz 2011)), and it can be easily related to the formulation in Eq. (1). Assuming the signals $x$ and $y$ are random variables, the relative exponent of the PDF metric is given as follows:

$$
A_{\mathrm{pdf}}=D_{B}(x, y)=-\ln \int_{-\infty}^{\infty} \sqrt{\hat{p}_{x_{n}}(z) \hat{p}_{y_{n}}(z)} \mathrm{d} z .
$$

where $\hat{p}_{x_{n}}$ and $\hat{p}_{y_{n}}$ are estimates of the PDFs of the standardized signals (standard score) $x_{n}$ and $y_{n}$, respectively. For $\lambda_{\mathrm{PDF}}=1$, the PDF metric yields the following: 


$$
M_{\mathrm{pdf}}=\int_{-\infty}^{\infty} \sqrt{\hat{p}_{x_{n}}(z) \hat{p}_{y_{n}}(z)} \mathrm{d} z=B C\left(x_{n}, y_{n}\right)
$$

where $B C\left(x_{n}, y_{n}\right)$ is the Bhattacharyya coefficient which can take values in the range $0 \leq B C \leq 1$, which corresponds with the constructed comparison metrics. Formulated as such, the PDF metric can be also related to Hellinger's distance or Rényi divergence (cf. e.g. Nielsen and Boltz (2011)).

In Eqs. (7) and (8), the standard scores of the signals are used to asses the discrepancies in the third and higher-order standardized moments of the PDF. This is to avoid redundancy in the metrics to some extent, as the first- and second-order moments are included in the RMS metric. The estimated PDFs, $\hat{p}_{x_{n}}$ and $\hat{p}_{y_{n}}$, are obtained by nonparametric kernel density estimation, utilizing the Gaussian kernel and the Improved Sheather-Jones algorithm for bandwidth selection (cf. Botev et al. (2010), including a матLAB code). Alternatively, the estimated PDFs can be modeled by parametric approaches utilizing, for e.g., Hermite polynomials (Gurley and Kareem 1997).

\section{Wavelet and frequency-normalized wavelet metrics}

The wavelet metric aims to quantify the discrepancies of the energy in the time-frequency plane of a signal. This is of significant importance in bridge aerodynamics as it reveals particular features of the aerodynamic forces and response, such as stationary and nonstationary frequency content, aerodynamic coupling, modal contribution, and local "bursts" of signal energy, etc. The interpretation in the timefrequency plane has been extensively utilized in wind engineering (cf. e.g. (Kareem and Kijewski 2002; Gurley et al. 2003)). Formulated as a metric, the wavelet transform has been used on several occasions in mechanics (cf. e.g. (Jiang and Mahadevan 2010; Teferra et al. 2014; Kristeková et al. 2009)).

To obtain the time-frequency representation, the wavelet transform of a signal $x(t)$ is given as follows (Grossmann and Morlet 1984):

$$
W_{x}(a, t)=\frac{1}{\sqrt{a}} \int_{-\infty}^{\infty} x(\tau) \psi^{*}\left(\frac{t-\tau}{a}\right) \mathrm{d} \tau
$$

where $W_{x}$ are complex wavelet coefficients, $a$ is the scale parameter, $\psi=\psi(t)$ is the mother wavelet, and the asterisk "*" denotes the complex conjugate. Herein, the Morlet wavelet, normalized with respect to the wavelet energy, is utilized and is given as follows (cf. e.g. Jamšek et al. (2007)):

$$
\psi(t)=\pi^{-1 / 4} e^{-i 2 \pi f_{0} t} e^{-t^{2} / 2},
$$

where $i=\sqrt{-1}$ is the imaginary unit, while $f_{0}$ is the central frequency of the wavelet which is crucial for defining the time and frequency resolution and should be adjusted accordingly (Kijewski and Kareem 2003). For the Morlet wavelet, the Fourier frequency $f$ is related to the scale parameter as $f=f_{0} / a$. The squared magnitude of the wavelet coefficients (i.e. scalogram) reveals the frequencies where the energy is concentrated in a time-localized manner. As the interpretation in this study is based on the time-frequency instead of the time-scale representation, the wavelet coefficients are based on the frequency in the following.

The relative exponent of the wavelet metric is obtained as a normalized RMS deviation of the magnitude of the wavelet coefficients, and it yields the following:

$$
A_{w f}=\frac{\sqrt{\int_{0}^{T} \int_{0}^{\infty}\left[\left|W_{x}(f, t)\right|-\left|W_{y}(f, t)\right|\right]^{2} \mathrm{~d} f \mathrm{~d} t}}{\sqrt{\int_{0}^{T} \int_{0}^{\infty}\left[\left|W_{x}(f, t)\right|\right]^{2} \mathrm{~d} f \mathrm{~d} t}} .
$$

By obtaining the wavelet metric in such a way, the discrepancies between the two signals in the time-frequency plane are quantified. From a practical aspect, the wavelet parameters such as the mother wavelet, central 
wavelet frequency, and the frequency range should remain the same for both signals. Moreover, for the wavelet-based metrics, the integration of the time-frequency plane should encompass an area that neglects the wavelet end effects, commonly referred to as the cone of influence (Kijewski and Kareem 2003).

The wavelet metric incorporates the total discrepancy in the time-frequency plane. To further study whether this discrepancy is due to the amplitude or frequency difference, the frequency-normalized wavelet metric is introduced by normalizing the instantaneous wavelet amplitude with respect to the frequency marginal. The relative exponent for this metric is given as follows:

$$
A_{w f}=\frac{1}{T} \int_{0}^{T} \frac{\sqrt{\int_{0}^{\infty}\left[\frac{\left|W_{x}(f, t)\right|}{W_{x f, \max }(t)}-\frac{\left|W_{y}(f, t)\right|}{W_{y f, \max }(t)}\right]^{2} \mathrm{~d} f}}{\sqrt{\int_{0}^{\infty}\left[\frac{\left|W_{x}(f, t)\right|}{W_{x f, \max }(t)}\right]^{2} \mathrm{~d} f}} \mathrm{~d},
$$

where the normalization factor, for e.g., for signal $x(t)$ is given by the following:

$$
W_{x f, \max }(t)=\max _{f}\left|W_{x}(t, f)\right| .
$$

Practically, this metric quantifies the distortion in the shape of the local spectrum, which is of particular interest for aerodynamic models resulting in multi-frequency output for single-frequency input. A metric normalized with respect to the magnitude marginal can be constructed in a similar fashion; however, this metric is not considered herein, since it is deemed to be a redundant addition to the magnitude metric.

\section{Stationarity metric}

A stationarity metric is introduced herein to detect, isolate and quantify the difference in the nonstationary portion in the time-frequency plane of the signals. This metric aims to capture the nonstationarity amplitude and frequency modulations in the aerodynamic forces for large angles of attack. The relative exponent is formulated as:

$$
A_{s}\left\{\begin{array}{lll}
\rightarrow \infty & \text { if } & \Theta(x) \neq \Theta(y), \\
=0 & \text { if } & \Theta(x)=\Theta(y)=0, \\
=\frac{\sqrt{\int_{0}^{T} \int_{0}^{\infty}\left[\left|W_{x}^{F}(f, t)\right|-\left|W_{y}^{F}(f, t)\right|\right]^{2} \mathrm{~d} f \mathrm{~d} t}}{\sqrt{\int_{0}^{T} \int_{0}^{\infty}\left[\left|W_{x}^{F}(f, t)\right|\right]^{2} \mathrm{~d} f \mathrm{~d} t}} & \text { if } & \Theta(x)=\Theta(y)=1 .
\end{array}\right.
$$

where $\Theta$ is a binary indicator of signal nonstationarity, while $W^{F}(f, t)$ represents the filtered nonstationary part of the wavelet coefficients, if the signal is determined to be nonstationary. To determine whether the signal is nonstationary and if so, to filter the nonstationary part of the wavelet coefficients, a local and a global method (cf. Borgant et al. (2010), McCullough and Kareem (2013), respectively) based on surrogates and hypothesis testing are utilized. In the following, these methods are briefly revisited and combined to facilitate the two-step procedure in Eq. (14).

A surrogate of a signal is obtained by manipulating the original time-histories to satisfy the null hypothesis while retaining most of the properties such as the power spectrum or PDF. Since the null hypothesis in this case is that the signal is stationary, the Fourier surrogates have shown to be adequate (Richard et al. 2010; McCullough and Kareem 2013). A Fourier surrogate of a signal $x(t)$ is obtained by randomizing the phase 
of its Fourier transform $X(f)$ while retaining the modulus at each frequency and then applying the inverse Fourier transform. This yields the following:

$$
x_{\text {sur }}(t)=\int_{-\infty}^{\infty} e^{i 2 \pi f t}|X(f)| e^{i \phi_{R}} \mathrm{~d} f
$$

where $\phi_{R}$ is a random phase, uniformly distributed over the interval $[-\pi, \pi)$.

The first step is to determine whether the signal is stationary by comparing it to surrogate signals utilizing discriminating statistics. The deterministic statistics are based on the statistical distance between the local and global spectrum. If the discriminating statistic of the original signal $\sigma_{x}$ falls out of the one-sided confidence interval of the probability density of the discriminating statistic of the surrogates $\sigma_{\text {sur }}$, the null hypothesis is rejected for the original signal. This is formulated as follows:

$$
\Theta(x)=\left\{\begin{array}{llll}
0 & \text { if } & p\left(\sigma_{\text {sur }} \leq \sigma_{x}\right) \leq \gamma ; & \text { "stationary", } \\
1 & \text { if } & p\left(\sigma_{\text {sur }} \leq \sigma_{x}\right)>\gamma ; & \text { "nonstationary". }
\end{array}\right.
$$

where $\gamma$ corresponds to the confidence level. The discriminatory statistic $\sigma_{\text {sur }}$ is, in fact, a random variable for which an estimated parametric PDF is obtained, such as the Gaussian or Gamma density, for a number of surrogates. For a single time-history, the discriminatory statistic is obtained based on the divergence $D(t)$ between the local and global spectrum as follows:

$$
\sigma=\operatorname{Var}[D(t)]
$$

A combination of the Kullback-Leiber divergence and log-spectral deviation is utilized herein for the distance, since it has shown to be adequate for both cases of amplitude and frequency modulation (Borgant et al. 2010). This distance is given as follows:

$$
D_{\mathrm{LSD}+\mathrm{KL}, x}(t)=\left\{\int_{0}^{\infty}\left[S_{x, n}(t, f)-\overline{S_{x, n}}(f)\right] \log \frac{S_{x, n}(t, f)}{\overline{S_{x, n}}(f)} \mathrm{d} f\right\} \times\left\{1+\int_{0}^{\infty}\left|\log \frac{S_{x}(f, t)}{\overline{S_{x}}(f)}\right| \mathrm{d} f\right\},
$$

where the $S_{x}(f, t)$ and $\bar{S}_{x}(f)$ are the scalogram and global spectrum, which are obtained as follows:

$$
\begin{gathered}
S_{x}(f, t)=\left|W_{x}(f, t)\right|^{2}, \\
\overline{S_{x}}(f)=\frac{1}{T} \int_{0}^{T} S_{x}(f, t) \mathrm{d} t,
\end{gathered}
$$

and $S_{x, n}(t, f)$ and $\overline{S_{x, n}}(f)$ are the corresponding normalized versions of the scalogram and global spectrum, respectively.

In the second step, if a signal is determined to be nonstationary (cf. Eq. 16), the nonstationary part of the wavelet coefficients $W_{x}^{F}(f, t)$ are filtered using a threshold scalogram based on stationary surrogates (McCullough and Kareem 2013). This yields the following:

$$
W_{x}^{F}(f, t)=\left\{\begin{array}{lll}
0 & \text { if } & S_{x}(f, t) \leq \max _{t} S_{\mathrm{tr}, \mathrm{x}}(f, t) \\
W_{x}(f, t) & \text { if } & S_{x}(f, t)>\max _{t} S_{\mathrm{tr}, \mathrm{x}}(f, t)
\end{array}\right.
$$

where $S_{t r, x}(f, t)$ is a threshold scalogram. Herein, the maximum threshold scalogram is considered with respect to the time marginal instead of a localized value as in McCullough and Kareem (2013), since the threshold peak can occur at any time, under the stationarity assumption. The threshold, assuming normality, 
is defined at each point on the time-frequency plane by using the mean $S_{\mathrm{sur}, M x}(f, t)$ and standard deviation $S_{\mathrm{sur}, S D x}(f, t)$ of the surrogate scalograms as follows:

$$
S_{\mathrm{tr}, \mathrm{x}}(f, t)=S_{\mathrm{sur}, M x}(f, t)+g S_{\mathrm{sur}, S D x}(f, t),
$$

where $g$ is a factor depending on the probability of exceeding the threshold.

It should be noted that it can be determined whether the signal is stationary only by using the local method in Eq. (21); hence, the global method in the first step (cf. Eq. (16)) can be considered redundant. However, herein the two-step procedure is utilized as the computation of the standard deviation of the surrogate scalograms in Eq. (22) requires either retaining all surrogate scalograms in computer memory or their re-computation once $S_{\mathrm{sur}, M}(f, t)$ is determined. Hence, utilizing the two-step procedure can help reducing computational memory when obtaining $M_{s}$ for stationary signals. Moreover, to filter the complete stationary part of the signal for finely discretized time marginal, a large number of surrogate is required. Otherwise, local spurious peaks may be still present in the filtered scalogram, which would result in a sensitive stationary metric. Detecting stationarity with a global binary test, followed by a local quantification of the nonstationary parts has shown to alleviate this problem for a reasonable number of surrogates.

\section{Bispectrum metric}

The sub- and superharmonics are distinctive nonlinear features in signals and they appear as a result of the nonlinear interaction between harmonics. Higher-order spectra have been proven to be a useful tool for detection and modeling of such nonlinearities in mechanical and phyisical systems (cf. e.g. (Fackrell et al. 1995; Nikitas and Petropulu 1993; Kim and Powers 1979; van Milligen et al. 1995)). Wu and Kareem (2013a) utilized the Fourier bispectrum to study the quadratic nonlinear interaction between two frequencies (i.e. quadratic phase coupling) for experimental signals of aerodynamic forces acting on a bridge deck at high angles of attacks.

Herein, the bispectrum metric is formulated based on the wavelet bispectrum including a quadratic phase coupling detector. The main advantage of utilizing the wavelet instead of the Fourier bispectrum is that it can detect intermittent nonlinearities and the statistical error is reduced (Jamšek et al. 2007). Moreover, the quadratic phase coupling detector offers detection of quadratic nonlinearities, which is particularly useful for synthetic signals (Fackrell and McLaughlin 1995). With this, the bispectrum metric aims to first identify and then quantify the discrepancies in the quadratic aerodynamic nonlinearity.

The wavelet bispectrum $B_{w x}\left(f_{1}, f_{2}\right)$ of a signal $x(t)$ is obtained from the wavelet coefficients (cf. Eq. (9)) as follows (van Milligen et al. 1995):

$$
B_{w x}\left(f_{1}, f_{2}\right)=\int_{T_{B}} W_{x}\left(f_{1}, \tau\right) W_{x}\left(f_{2}, \tau\right) W_{x}^{*}\left(f_{3}, \tau\right) \mathrm{d} \tau,
$$

where $f_{3}=f_{1}+f_{2}$ and $T_{B}$ is the integration interval of interest. Since the wavelet bispectrum is a complex quantity, it can be expressed in terms of amplitude $G_{B x}\left(f_{1}, f_{2}\right)$ and phase $\phi_{B x}\left(f_{1}, f_{2}\right)$ as follows:

$$
B_{w x}\left(f_{1}, f_{2}\right)=G_{B x}\left(f_{1}, f_{2}\right) e^{i \phi_{B x}\left(f_{1}, f_{2}\right)} .
$$

The instantaneous biphase can be obtained from Eqs. (23) and (24) as follows:

$$
\phi_{B x}\left(f_{1}, f_{2}, t\right)=\phi\left(f_{1}, t\right)+\phi\left(f_{2}, t\right)-\phi\left(f_{3}, t\right) .
$$

Equation (23) can indicate whether there is nonlinear interactions (i.e. quadratic phase coupling) between the $f_{1}$ and $f_{2}$ harmonics, yielding nontrivial values of the bispectrum magnitude at the $f_{2}-f_{1}$ and $f_{2}+f_{1}$ frequency couples. In addition to nontrivial magnitude at these frequency pairs, the condition $\phi_{B x}=0$ for the biphase in Eq. (25) is necessary to be satisfied for quadratic phase coupling to occur (Fackrell and McLaughlin 
1995). If this condition is not satisfied, the normalized bispectrum, i.e. bicoherence (Fourier- or wavelet), can yield in nontrivial value despite the absence of nonlinearities in the signal. This is particularly true for synthetically generated signals with a constant phase, such as broadband forces/response, for which the bispectrum can have multiple peaks corresponding to the discrete frequencies or natural signal frequencies. Therefore, further adjustment of the bispectrum is required to detect the quadratic nonlinearity.

In this study, a modified quadratic-phase coupling detector is utilized, based on the phase-randomized bispectrum introduced by Kim et al. (2007) for the Fourier, and by Li et al. (2009) and Scully et al. (2017) for the wavelet bispectrum. The phase-randomized bispectrum $B_{w x}^{R}\left(f_{1}, f_{2}\right)$ is obtained as follows

$$
B_{w x}^{R}\left(f_{1}, f_{2}\right)=\int_{T_{B}} G_{B x}\left(f_{1}, f_{2}, \tau\right) e^{i R_{B}\left(f_{1}, f_{2}, \tau\right) \phi_{B x}\left(f_{1}, f_{2}, \tau\right)} \mathrm{d} \tau,
$$

where $R_{B}\left(f_{1}, f_{2}, \tau\right)$ is a uniformly distributed random phase in the interval $\left[-r_{B}, r_{B}\right)$, where $r_{B}$ is a randomization factor. From Eq. (26), it can be deduced that if $\phi_{B x}\left(f_{1}, f_{2}, t\right)=0$, the exponential term amounts to 1 , while, if $\phi_{B x}\left(f_{1}, f_{2}, t\right) \neq 0$, the random value $R_{B}\left(f_{1}, f_{2}, t\right)$ can considerably reduce the bispectrum. This reduction depends on the randomization factor $r_{B}$. In previous studies (cf. e.g. Scully et al. (2017)) this factor is taken as $r_{B}=\pi$; however, this does not offer insight of how the phase-randomized bispectrum behaves. Considering a constant bispectrum amplitude $G_{B x}\left(f_{1}, f_{2}\right)$ and constant biphase $\phi_{B x}\left(f_{2}, f_{2}\right)$, which is not necessarily zero, the influence of the $r_{B}$ factor on the resultant phase-randomized bispectrum amplitude $G_{B x}^{R}$ is shown in Fig. 6. It can be seen that the larger $r_{B}$ is, the faster $G_{B x}^{R}$ attenuates towards zero for biphase angles deviating from zero. Moreover, there is a "ripple" effect after $G_{B x}^{R}$ reaches zero. Theoretically, there should be no deviation for $\phi_{B x}$ from zero in case of quadratic phase coupling; however, noise can affect the bispectrum (Elgar and Sebert 1989) and thus, the angle $\phi_{B x}$ can take small values. Although it would be the most appropriate to select the $r_{B}$ factor based on the level of noise, in this study this factor is selected as $r_{B}=10 \pi$. This corresponds to an angle of $\phi_{B x}=2 \pi / r_{B}=0.2 \mathrm{rad}$, for which it is assumed that quadratic phase coupling is present, i.e. when $G_{B x}^{R}$ first intercepts the abscissa (cf. Fig. 6).

The phase-randomized bispectrum is still affected by noise and, additionally, due to the ripple effect of the biphase randomization. In addition to a zero biphase, i.e. $\phi_{B x}=0$, the amplitude of $G_{B x}^{R}$ should be also significant to indicate that the quadratic phase coupling between two harmonics is not due to noise (Fackrell and McLaughlin 1995). In order to obtain statistically significant estimation of the $B_{w x}^{R}$, a surrogate method can be employed. The generation of surrogates can be conducted in similar fashion as for the stationarity metric, by manipulating the original signal properties. For the wavelet bispectrum, Fourier surrogates cannot be utilized as stationarity is not assumed; thus, more advanced surrogates should be utilized such as the wavelet iterative amplitude adjusted Fourier transform surrogates (cf. e.g. Lancester et al. (2018), McCullough (2016)). However, these surrogates are computationally very demanding; therefore,

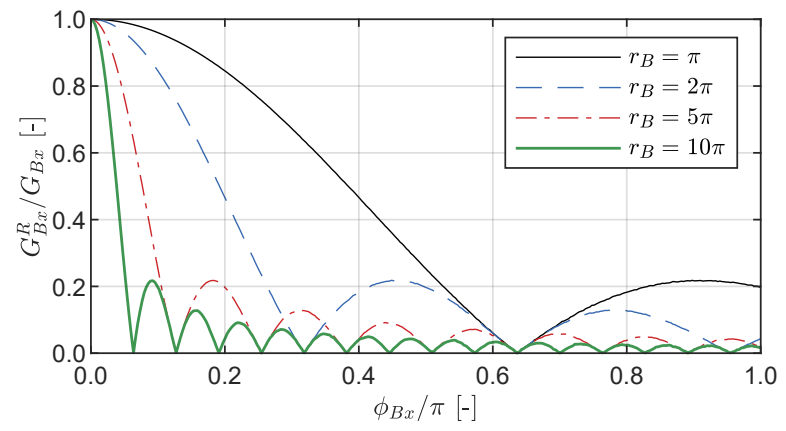

Fig. 6. Effect of the randomization factor $r_{B}$ on the ratio between the phase-randomized bispectrum magnitude $G_{B x}^{R}$ and bispectrum magnitude $G_{B x}$ for a constant biphase $\phi_{B x}$. 
the surrogate bispectrum map is obtained simply by eliminating the biphase information in the original bispectrum directly. This can be done by simply setting a uniformly distributed random biphase $\phi_{B R}\left(f_{1}, f_{2}\right)$ in the interval $\left[-\pi:-r_{\text {sur }}, r_{\text {sur }}: \pi\right)$, for $0<r_{\text {sur }}<\pi$, in Eq. (26) as follows:

$$
B_{w \text { sur }}^{R}\left(f_{1}, f_{2}\right)=\int_{T_{B}} G_{B x}\left(f_{1}, f_{2}, \tau\right) e^{i R_{k}\left(f_{1}, f_{2}, \tau\right) \phi_{B R}\left(f_{1}, f_{2}\right)} \mathrm{d} \tau .
$$

The null hypothesis for the surrogate bispectrum amplitude map is that the signal does not exhibit quadratic phase coupling. Hence, the biphase $\phi_{B R}\left(f_{1}, f_{2}\right)$ should not amount to zero. This is controlled by the random factor $r_{\text {sur }}$. In Li et al. (2009), the random biphase $\phi_{B R}$ is obtained by adding to the initial biphase $\phi_{B x}$ a time-varying random phase within the $[\pi, \pi)$ interval. In this study, $\phi_{B R}$ is taken to be constant in time as it is a more critical case for which the bispectrum can result in false detection of nonlinearities. This creates bispectrum surrogate map which is based to the original phase-randomized bispectrum $B_{w x}^{R}$ and additionally ensuring that the biphase $\phi_{B x}$ does not amount to zero by using the factor $r_{\text {sur. Since the factor }}$ $r_{B}$ assures quadratic phase coupling, the factor $r_{\text {sur }}$ should be a complement in a way; hence, it is obtained as $r_{\text {sur }}=2 \pi / r_{B}$. In this manner, the wavelet bispectrum is filtered for a nonzero biphase and an insignificant amplitude.

Similarly as for the stationarity metric, the threshold bispectrum amplitude map $G_{\mathrm{Btr}}^{R}\left(f_{1}, f_{2}\right)$ can be obtained as follows:

$$
G_{B \operatorname{tr}, x}^{R}\left(f_{1}, f_{2}\right)=G_{B \operatorname{sur}, M x}^{R}\left(f_{1}, f_{2}\right)+g G_{B \operatorname{sur}, S D x}^{R}\left(f_{1}, f_{2}\right),
$$

where $G_{B \text { sur }, M x}^{R}\left(f_{1}, f_{2}\right)$ and $G_{B \text { sur }, S D x}^{R}\left(f_{1}, f_{2}\right)$ are the mean and standard deviation of the surrogate bispectrum amplitude, respectively. These are obtained for a number of surrogates of the surrogate phase-randomized bispectrum amplitude $G_{\text {sur }}^{R}\left(f_{1}, f_{2}\right)$ (based on Eq. (27)), while $g$ is a factor depending on the probability of exceeding the threshold.

The filtered phase-randomized bispectrum amplitude $G_{W x}^{F}$ can be obtained from the phase-randomized bispectrum amplitude $G_{B x}^{R}\left(f_{1}, f_{2}\right)$ as follows:

$$
G_{B x}^{F}\left(f_{1}, f_{2}\right)=\left\{\begin{array}{lll}
0 & \text { if } & G_{B x}^{R}\left(f_{1}, f_{2}\right) \leq \max G_{B \mathrm{tr}, x}^{R}\left(f_{1}, f_{2}\right), \\
G_{B x}^{R}\left(f_{1}, f_{2}\right) & \text { if } & G_{B x}^{R}\left(f_{1}, f_{2}\right)>\max G_{B \mathrm{tr}, x}^{R}\left(f_{1}, f_{2}\right) .
\end{array}\right.
$$

Although the noise level is frequency dependent (van Milligen et al. 1995), the maximum value is taken for filtering as threshold, since spurious peaks could remain for finely discretized frequencies. This would results in a very sensitive bispectrum metric. Moreover, the bispectrum for signals without quadratic phase coupling should be zero anyway. Alternatively, the values at each individual frequency pair can be taken; however, this requires larger number of surrogates, which is of high computational cost.

Finally, the relative exponent for the wavelet bispectrum metric is obtained as follows:

$$
A_{b}\left\{\begin{array}{llll}
\rightarrow \infty & \text { if } & \Gamma(x) \neq \Gamma(y), \\
=0 & \text { if } & \Gamma(x)=\Gamma(y)=0, \\
=\frac{\sqrt{\int_{0}^{\infty} \int_{0}^{\infty}\left[G_{B x}^{F}\left(f_{1}, f_{2}\right)-G_{B y}^{F}\left(f_{1}, f_{2}\right)\right]^{2} \mathrm{~d} f_{1} \mathrm{~d} f_{2}}}{\sqrt{\int_{0}^{\infty} \int_{0}^{\infty}\left[G_{B x}^{F}\left(f_{1}, f_{2}\right)\right]^{2} \mathrm{~d} f_{1} \mathrm{~d} f_{2}}} & \text { if } & \Gamma(x)=\Gamma(y)=1,
\end{array}\right.
$$

where $\Gamma$ indicates whether there is a quadratic phase coupling in a signals and is given by the following:

$$
\Gamma(x)=\left\{\begin{array}{llll}
0 & \text { if } & \max G_{B x}^{F}\left(f_{1}, f_{2}\right)=0 ; & \text { "linear", } \\
1 & \text { if } & \max G_{B x}^{F}\left(f_{1}, f_{2}\right)>0 ; & \text { "nonlinear". }
\end{array}\right.
$$


From Eq. (30), it can be gathered that the bispectrum metric quantifies the discrepancies in the nonlinear portion of the two time-histories, should both contain quadratic phase coupling. In practice, the integration in Eq. (30) is only about the part of the bispectrum that is encompassed by the inner triangle (cf. e.g. Fackrell et al. (1995)), to avoid the redundant part of the bispectrum.

\section{PERFORMANCE EVALUATION OF THE COMPARISON METRICS}

In this section, the performance of the comparison metrics is assessed by conducting controlled numerical experiments using generic signals. The goal is to track the performance of the metrics using known signal features. This ultimately reveals the sensitivity of specific metrics to specific signal feature changes. The matlab code, including the results from this section, is freely available and is deposited in an online respiratory (see Data Availability Statement). Four generic signals, $x_{i}(t)$ for $i=\{1, \ldots, 4\}$, are studied with respect to a reference one $x_{r}(t)$. The considered reference signal is given as follows:

$$
x_{r}(t)=A_{1} \cos \left(\omega_{1} t\right)+A_{2} \cos \left(\omega_{2} t+\frac{\pi}{3}\right)+\eta_{r}(t) .
$$

The generic signals are labeled corresponding to the particular signal feature that is being modified as follows:

- Signal 1 - Phase shift

$$
x_{1}(t)=A_{1} \cos \left(\omega_{1} t+\phi_{1}\right)+A_{2} \cos \left(\omega_{2} t+\frac{\pi}{3}+\phi_{2}\right)+\eta_{1}(t)
$$

- Signal 2 - Amplitude scaling

$$
x_{2}(t)=2 A_{1} \cos \left(\omega_{1} t\right)+2 A_{2} \cos \left(\omega_{2} t+\frac{\pi}{3}\right)+\eta_{2}(t)
$$

- Signal 3 - Frequency modulation

$$
x_{3}(t)=A_{1} \cos \left(\omega_{1} t\right)+A_{2} \cos \left(\omega_{2} t+K t^{2}+\frac{\pi}{3}\right) e^{-\lambda_{1} t}+\eta_{3}(t)
$$

- Signal 4 - Nonlinearity

$$
x_{4}(t)=A_{1} \cos \left(\omega_{1} t\right)+A_{2} \cos \left(\omega_{2} t+\frac{\pi}{3}\right)+\frac{A_{1}+A_{2}}{2} \cos \left(\omega_{1} t\right) \cos \left(\omega_{2} t+\phi_{3}\right) e^{-\lambda_{2} t}+\eta_{4}(t) .
$$

The following parameters are considered: $A_{1}=1, A_{2}=1.3, \omega_{1}=2 \times 2 \pi, \omega_{1}=2.8 \times 2 \pi, \phi_{1}=\pi$, $\phi_{2}=\pi / 6, K=\left(\omega_{3}-\omega_{2}\right) / T, \omega_{3}=3.6 \times 2 \pi, \phi_{3}=\pi / 3, T=100$ s is the signal length with sampling frequency of $f_{s}=100 \mathrm{~Hz}$, and $\eta(t)$ is a white noise with zero mean for signal-to-noise ratio amounting to 10. Initially, the parameters are set $\lambda_{1}=\lambda_{2}=0$; however, this is modified later when attempting to quantify the discrepancies in the nonlinear and nonstationary parts. For computation of the comparison metrics the following parameters are selected: normalization time $T_{c}=2 \pi / \omega_{2}$, wavelet central frequency $f_{0}=6 \mathrm{~Hz}$, confidence level for the discriminating statistic $\gamma=95 \%$, and surrogate map factor $g=2$. A total of 200 surrogates are deemed sufficient for the local stationarity analysis (Borgant et al. 2010), while 100 surrogates for the surrogate bispectrum map (Scully et al. 2017). Due to the dynamic time warping, the number of signal time-steps increased for the magnitude metric, resulting in a ratio of warped to original number of time-steps of $N_{w} / N=\{1.23,1.55,1.41,1.45\}$ for the generic signals $x_{j}$ for $j=\{1, \ldots, 4\}$, respectively. As an example, the original and warped signals for $x_{r}$ and $x_{1}$ are shown in Fig. 4. The resulting comparison metrics are given in Fig. 7, while in Fig. 8, the wavelet coefficients for all the signals are given for all signals except $x_{1}$, since they are similar as for $x_{r}$. The following discussion is based on these two figures. 

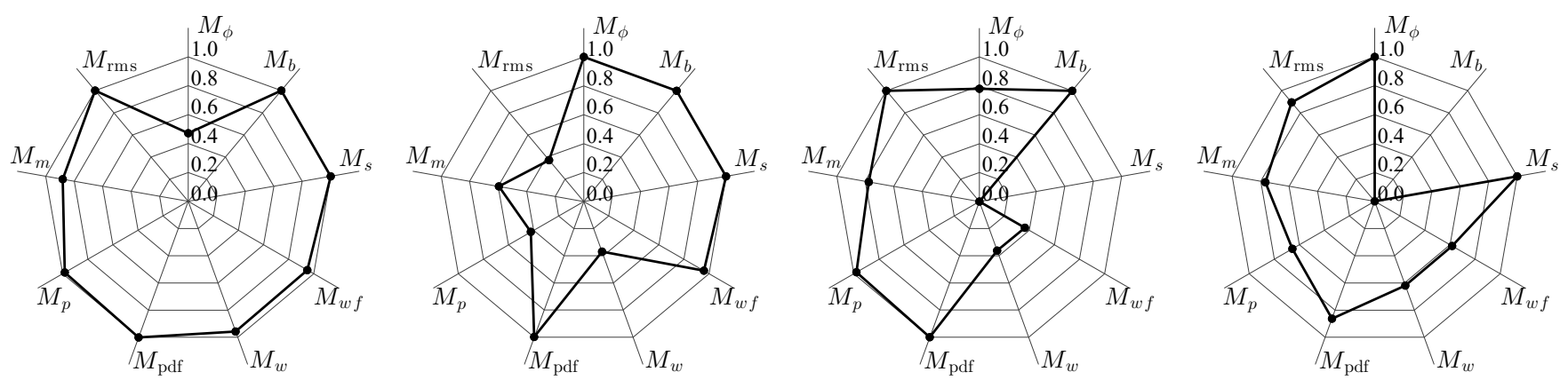

Fig. 7. Comparison metrics for the generic signals. From left to right: Phase Shift $M^{x_{r}, x_{1}}$, Amplitude Scaling $M^{x_{r}, x_{2}}$, Frequency Modulation $M^{x_{r}, x_{3}}$, and Nonlinearity $M^{x_{r}, x_{4}}$.
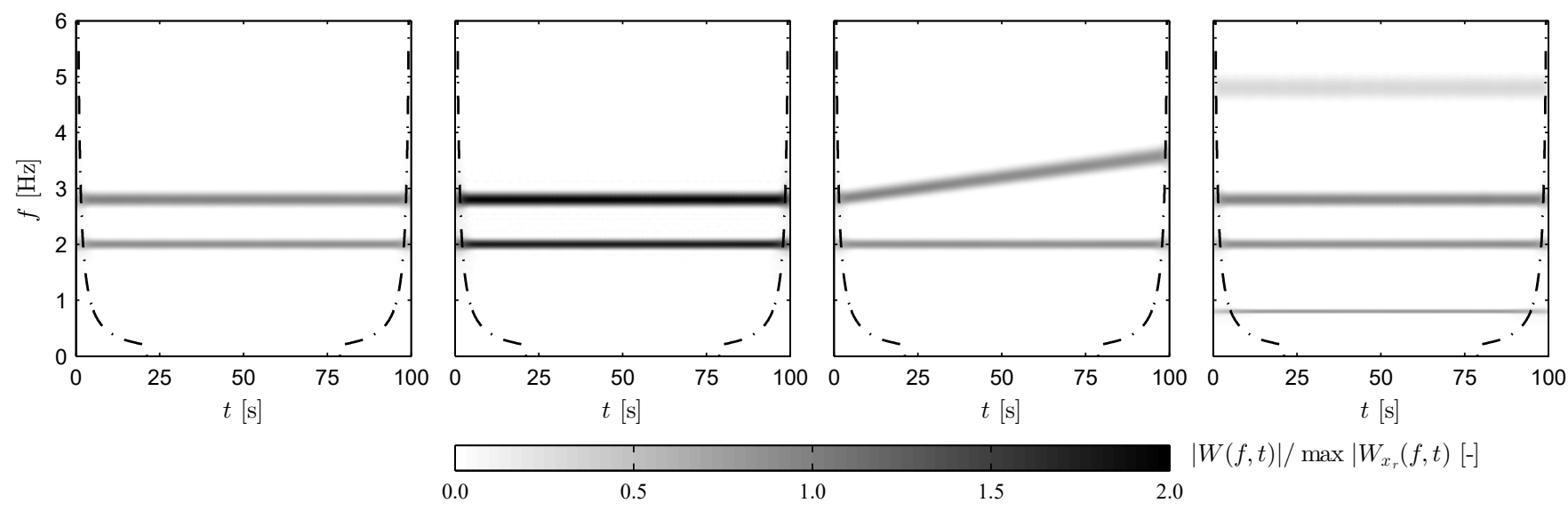

$|W(f, t)| / \max \mid W_{x_{r}}(f, t)[-]$

Fig. 8. Wavelet amplitude $|W(f, t)|$ for the generic signals. From left to right: $W_{x_{r}}, W_{x_{2}}, W_{x_{3}}$, and $W_{x_{4}}$. The dashed-dot line indicates the cone of influence.

\section{Signal 1 - Phase shift}

The first example is constructed to study the effect of phase shift since a time-lag is a common manifestation of the fluid memory in unsteady aerodynamic models. As expected, the phase metric $M_{\phi}^{x_{r}, x_{1}}$ results in a lower value (cf. Fig. 7, left). Moreover, the magnitude metric obtained using the warped signals results in a value of $M_{m}^{x_{r}, x_{1}}=0.88$. For comparison, this metric amounts to a value of 0.27 for unwarped signals, which is unrealistic as the signals are only phase-shifted. Due to the added noise, the wavelet metrics amount to $M_{w}^{x_{r}, x_{1}}=M_{w f}^{x_{r}, x_{1}}=0.96$, which is slightly less than 1 due to the added noise. The signals $x_{r}$ and $x_{1}$ are similar considering the features accounted in the remaining comparison metrics.

\section{Signal 2 - Amplitude scaling}

Analyzing the second example, the behavior of the comparison metrics due to amplitude discrepancy is observed. The amplitude of the signal $x_{2}$ is two times larger than $x_{r}$. Increasing the amplitude in such way, affects the RMS $M_{\mathrm{rms}}^{x_{r}, x_{2}}$, magnitude $M_{m}^{x_{r}, x_{2}}$, peak $M_{p}^{x_{r}, x_{2}}$, and wavelet metric $M_{\mathrm{w}}$ (cf. Fig. 7). With the exception of the magnitude metric, the rest of the metrics amount to $M_{\mathrm{rms}}^{x_{r}, x_{2}} \approx M_{\mathrm{p}}^{x_{r}, x_{2}} \approx M_{\mathrm{w}}^{x_{r}, x_{2}} \approx 0.4$, which is logical as a discrepancy of $100 \%$ yields $e^{-1} \approx 0.37$. The magnitude metric resulted in a higher value due to the dynamic time warping. Another particularity is that the value of the frequency-normalized wavelet metric is $M_{w f}^{x_{r}, x_{2}} \approx 1$. This is due to the fact that both signals have the same relative frequency content, as it can be seen from the absolute value of the wavelet coefficients in Fig. 8. As anticipated, no discrepancies are detected 
by the phase, PDF, stationarity and bispectrum metrics, i.e. $M_{\phi}^{x_{r}, x_{2}}=M_{\mathrm{pdf}}^{x_{r}, x_{2}}=M_{s}^{x_{r}, x_{2}}=M_{b}^{x_{r}, x_{2}}=1$.

Signal 3 - Frequency modulation

The third example is devised to investigate the effect of frequency modulation. Practically, the signal $x_{3}$ contains a linear chirp, which can also be observed in the wavelet coefficients (cf. Fig. 8). Since the stationarity null hypothesis (cf. Eq. (16)) is accepted for $x_{r}$ and rejected for $x_{3}$, the stationarity metric amounts to $M_{s}^{x_{r}, x_{3}}=0$ (cf. Fig 7, center-right). The magnitude $M_{m}^{x_{r}, x_{3}}$ and wavelet-based metrics, $M_{w}^{x_{r}, x_{3}}$ and $M_{w f}^{x_{r}, x_{3}}$, result in substantially lower values than 1 , while the peak and RMS metrics are $M_{p}^{x_{r}, x_{3}}=M_{\mathrm{rms}}^{x_{r}, x_{3}} \approx 1$. This further reinforces the claim why multi-criteria assessment is required beyond discussions based on averaged or global features of the signals. Unlike the previous example, $M_{w f}^{x_{r}, x_{3}}$ has similar value as $M_{w}^{x_{r}, x_{3}}$, which brings the conclusion that the discrepancies in $M_{w}^{x_{r}, x_{3}}$ are due to relative frequency modulation, rather than amplitude modulation. Although the mean phase metric is $M_{\phi}^{x_{r}, x_{3}}<1$, quantifying the mean phase between a stationary and frequency modulated nonstationary signal is obsolete, as noted in the preceding section. The frequency modulation did not result in appritiable deviation of the PDF; hence, $M_{\mathrm{pdf}}^{x_{r}, x_{3}} \approx 1$.

To further investigate the stationarity metric for two nonstationary signals, the frequency-modulated harmonic in Eq. (35) is additionally damped by a factor $\lambda_{1}=0.025$ and the resulting signal is denoted as $x_{3}^{\prime}$. Figure 9 depicts the filtered nonstationary part of the absolute wavelet coefficients of the signals $x_{r}, x_{3}, x_{3}^{\prime}$, and the difference ||$W_{x_{3}}^{F}|-| W_{x_{3}^{\prime}}^{F}||$. It can be observed that there are some spurious peaks for the reference signal, despite the fact that the univariate test (cf. Eq. (16)) correctly accepted the null hypothesis. This is the reason why the two-level stationarity testing procedure is devised for the stationarity metric. For $x_{3}$ and $x_{3}^{\prime}$, the stationarity null hypothesis was rejected and the linear and damped linear chirp can be seen in the filtered wavelet coefficients (cf. Fig. 8). The discrepancy in the nonstationary part for $x_{3}$ and $x_{3}^{\prime}$ is quantified based on Eq. (30). Hence, the stationarity metric amounts to $M_{S}^{x_{4}, x_{3}^{\prime}}=0.48$ for these two signals.

\section{Signal 4 - Nonlinearity}

In the last example, the difference of the nonlinearity in terms of quadratic phase coupling is explored for signals $x_{r}$ and $x_{4}$. The wavelet bispectrum metric amounts to $M_{b}^{x_{r}, x_{4}}=0$ (cf. Fig. 7, right) since the reference signal is linear and $x_{4}$ is nonlinear due to multiplication of the harmonics at $\omega_{1}$ and $\omega_{2}$. The filtered wavelet bispectrum of $x_{r}$ is trivial (cf. Fig. 10, left), while the filtered bispectrum of $x_{4}$ is not (cf. Fig. 10, center-right). Hence, it is clear that the corresponding frequency couples at $\left(f_{1}, f_{2}\right)=(2,2.8-2)$ and $\left(f_{1}, f_{2}\right)=(2.8,2)$ are due to nonlinear interaction of $\omega_{1}$ and $\omega_{2}$ for the signal $x_{4}$. Moreover, the magnitude of
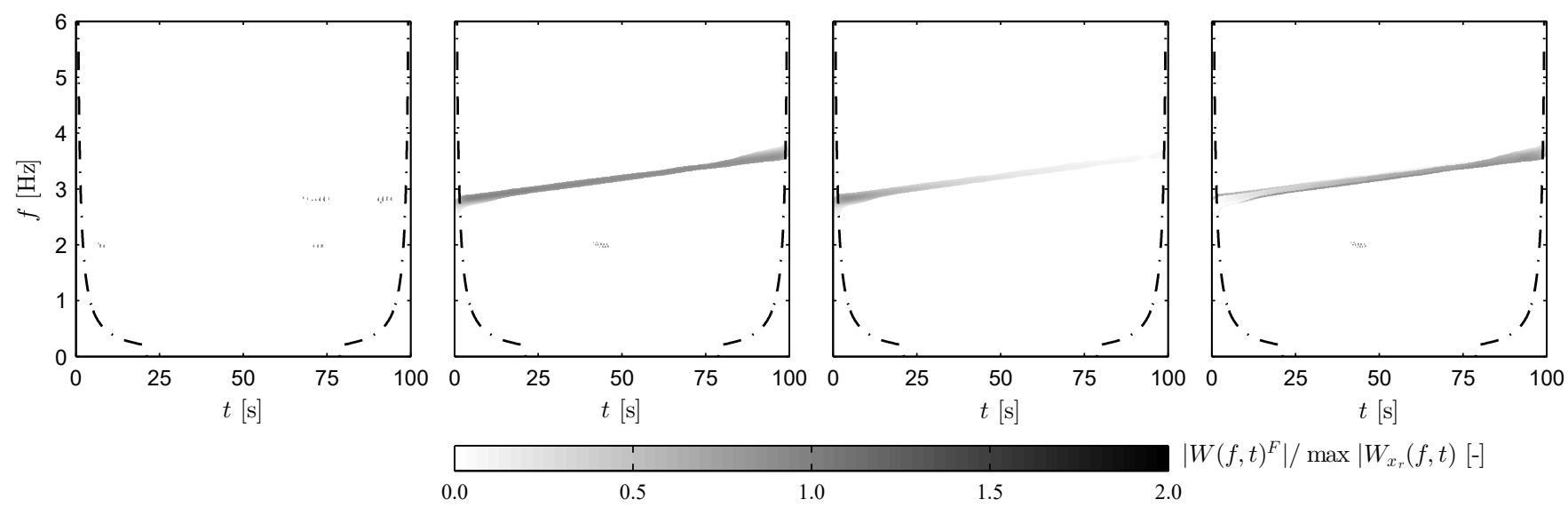

$W(f, t)^{F}|/ \max | W_{x_{r}}(f, t)[-]$

Fig. 9. Filtered nonstationary wavelet amplitude $\left|W^{F}(f, t)\right|$. for the generic signals. From left to right: $W_{x_{r}}^{F}$, $W_{x_{3}}^{F}, W_{x_{3}^{\prime}}^{F}$, and $W_{x_{3}}^{F}-W_{x_{3}^{\prime}}^{F}$. The dashed-dot line indicates the cone of influence. 
the wavelet coefficients indicates two additional frequencies at $0.8 \mathrm{~Hz}$ and $4.8 \mathrm{~Hz}$. Thus, most of the metrics involving signal amplitude comparison indicated discrepancies.

The filtering of the nonlinear part of the signal is demonstrated in Fig. 10, where the unfiltered (cf. Fig. 10, center-left) and filtered (cf. Fig. 10, center-right) wavelet bispectrum are depicted. It is clear that the additional noise of the bispectrum is removed by utilizing the presented surrogate method (cf. Eq. (29)). To quantify a discrepancy in the nonlinear portions of two signals, a signal $x_{4}^{\prime}$ is constructed based on Eq. (36) and by introducing a damping coefficient of $\lambda_{2}=0.025$. The wavelet bispectrum amplitude of $x_{4}^{\prime}$ is shown in Fig. 10 (right), and additionally, a "slice" of the wavelet bispectrum amplitude at $f_{2}=0.8 \mathrm{~Hz}$ is given in Fig. 11. Both of these figures indicate a difference in the wavelet bispectrum magnitude. Using Eq. (23) for the partial area of the inner triangle (separated by the white dashed-dot line in Fig. 10), the wavelet bispectrum metric amounts to $M_{b}^{x_{4}, x_{4}^{\prime}}=0.46$. The filtering threshold can be also observed in Fig. 11 . Further, by modifying $x_{4}$ in Eq. (36) for $\phi_{3}=0$, the filtered bispectrum amplitude resulted in zero values as the condition of $\phi_{B}=0$ is not satisfied. Hence, no quadratic phase coupling is detected. In this case, the filtered wavelet bispectrum is not shown for the sake of brevity as it is similar to for $x_{1}$ (cf. Fig. 10, left).

Furthermore, the PDF metric amounts to $M_{\mathrm{pdf}}^{x_{r}, x_{4}}=0.86$ for the last example, indicating a discrepancy in the PDFs. Figure 12 depicts the estimated PDFs of the normalized versions of the signals $x_{r}$ and $x_{4}$. It is
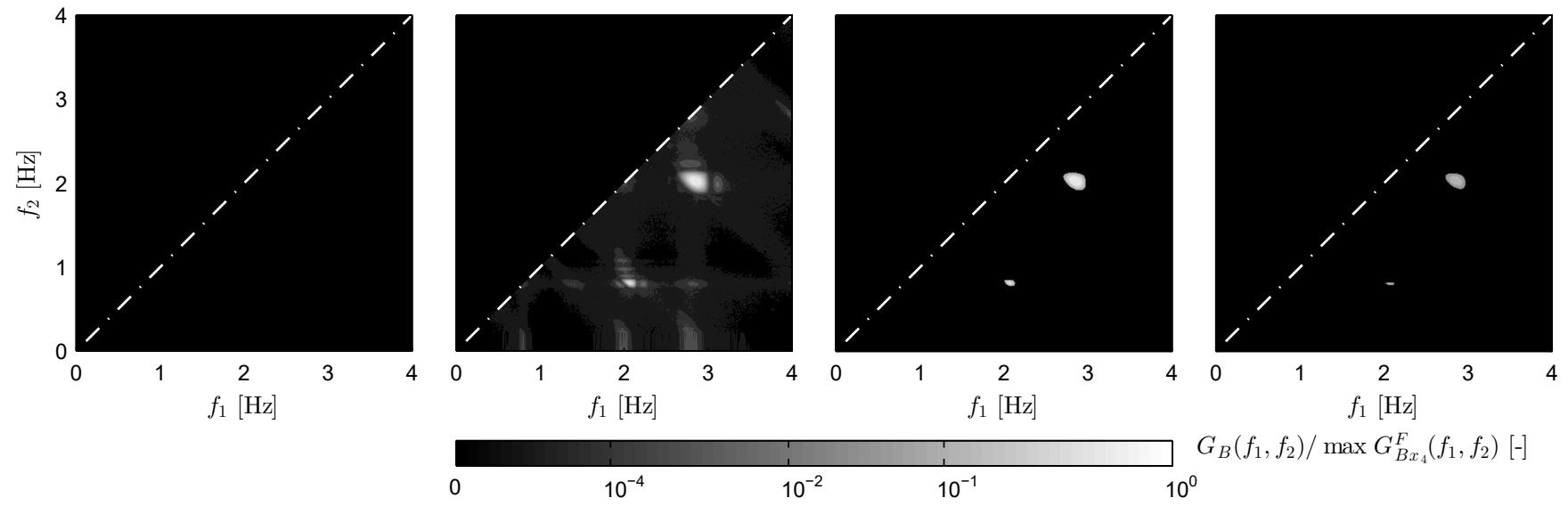

$G_{B}\left(f_{1}, f_{2}\right) / \max G_{B x_{4}}^{F}\left(f_{1}, f_{2}\right)[-]$ $10^{0}$

Fig. 10. Amplitude of the phase-randomized bispectrum $G_{B}^{R}\left(f_{1}, f_{2}\right)$ and filtered phase-randomized bispectrum $G_{B}^{F}\left(f_{1}, f_{2}\right)$ for the generic signals. From left to right: $G_{B x_{1}}^{F}, G_{B x_{4}}^{R}, G_{B x_{4}}^{F}$, and $G_{B x_{4}^{\prime}}^{F}$. The dashed-dot line indicates the inner triangle.

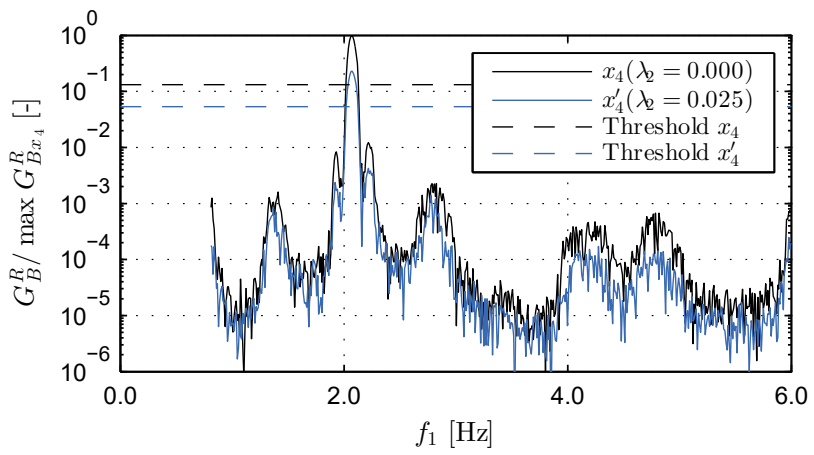

Fig. 11. Amplitude of the phase-randomized wavelet bispectrum $G_{B}^{R}\left(f_{1}, 0.8\right)$, including the filtering threshold. 


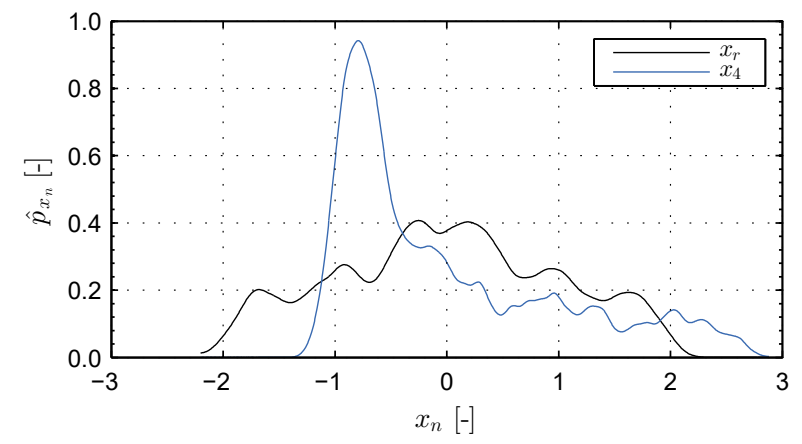

Fig. 12. Estimated PDF $\hat{p}$ of the normalized (standard score) signals $x_{r, n}$ and $x_{4, n}$.

apparent that the PDF of the signal $x_{4}$ is asymmetric, which is due to the quadratic part of Eq. (36). It can be argued that for such a large discrepancy in the estimated PDFs, the PDF metric should result in smaller values. This can accounted for be either by selecting another statistical divergence than the Bhattacharyya distance or by adjusting the sensitivity parameter $\lambda_{\text {pdf }}>1$ (cf. Eq. (1)). For e.g., $M_{\mathrm{pdf}}^{x_{r}, x_{4}}=0.74$ for $\lambda_{\mathrm{pdf}}=2$.

\section{APPLICATION TO BRIDGE AERODYNAMICS}

Having studied their behavior on generic signals in the preceding section, the comparison metrics are evaluated for an example from bridge aeroelasticity. The time-histories for the particular example utilized here are obtained from an extensive study by Kavrakov and Morgenthal (2018b) that deals with bridge aerodynamics. The aforementioned study entails a comparison of six semi-analytical aerodynamic models and a CFD model, based on the Vortex Particle Method, for aeroelastic analyses of a two-dimensional bridge deck at Reynold's number of $1.03 \times 10^{5}$. The Vortex Particle Method is a numerical method that uses discrete vortex particles to discretize the vorticity transport formulation of the Navier-Stokes equations for the fluid (cf. e.g. Cottet and Koumoutsakos (2000), Morgenthal and Walther (2007), Eldrege (2007), Akbari and Price (2003)) and has been utilized in bridge aerodynamics on numerous occasions (cf. e.g. Larsen and Walther (1997), Ge and Xiang (2008), Morgenthal et al. (2014)). The bridge deck of the Great Belt Bridge is selected, which is a streamlined box girder with a width of $B=31 \mathrm{~m}$ (cf. Fig. 13). Only a selected set of results is given herein, while detailed information on the model implementation, numerical discretization and results can be found in Kavrakov and Morgenthal (2018b). The comparison metrics are computed for the time-histories of two cases: (i) the aeroelastic response from buffeting analysis, and (ii) the self-excited forces under sinusoidal forced excitation. Unless specified differently, the parameters for the comparison metrics are the same as the ones described in the previous section.

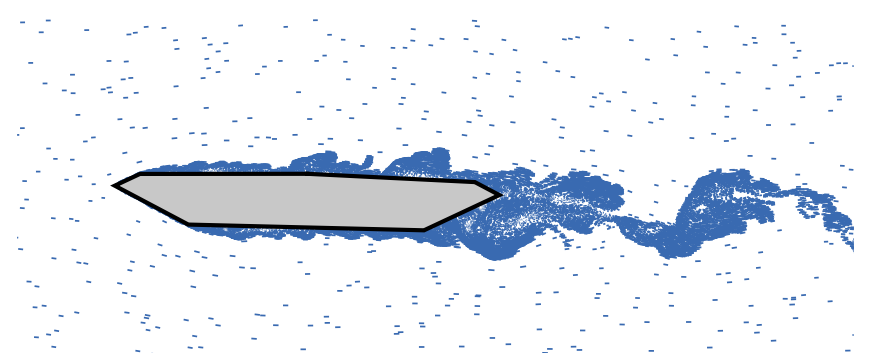

Fig. 13. Sample particle map of the bridge deck with turbulent free-stream (based on data from Kavrakov and Morgenthal 2018b). 


\section{Buffeting analysis}

The buffeting analysis is conducted for turbulent free-stream with mean wind speeds in the range of $U=20-60 \mathrm{~m} / \mathrm{s}$ and isotropic turbulence intensity of $6 \%$. For simplicity, the structural system is considered to have only a vertical and a torsional degree of freedom. Thus, the quantities of interest for the aeroelastic response are the time-histories of the vertical $h$ and rotational $\alpha$ displacements. The mass and moment of inertia of the moving deck components are set as $22.74 \mathrm{t} / \mathrm{m}$ and $2.47 \times 10^{3} \mathrm{tm}^{2} / \mathrm{m}$, respectively, which correspond to the first vertical and torsional frequencies amounting to $f_{h}=0.100 \mathrm{~Hz}$ and $f_{\alpha}=0.278 \mathrm{~Hz}$, respectively. The structural damping ratio is set as $0.5 \%$. Before subjecting the structure to the turbulent flow, a CFD simulation without a section is conducted in order to track the free-stream fluctuations at the location of the stiffness center of the section. The tracked fluctuations serve as an input for the semianalytical models, ensuring one-to-one comparison, i.e. all models are considered to have the same input free-stream turbulence. For the purpose of this study, only the Linear Unsteady (LU) (cf. e.g. Scanlan et al. (1974), Scanlan (2001), Caracoglia and Jones (2003)) and Quasi-steady (QS) (cf. e.g. Kovacs et al. (1992)) semi-analytical aerodynamic models are considered in addition to the CFD model. The LU model is linear and accounts for the fluid memory, while the QS model is nonlinear and disregards the fluid memory. Typically, the LU model is the standard semi-analytical model for aeroelastic analyses as the fluid memory effect is dominant and thus, the QS model is unsuitable; however, for complex deck shapes at high reduced velocity, aerodynamic nonlinearity can be prominent. Further information and interpretation of these assumptions is given in Kavrakov and Morgenthal (2018b), Wu and Kareem (2013b). The CFD model is taken as a reference as its mathematical formalism closer to fluid-structure interaction than both semi-analytical models (Kavrakov et al. 2019). However, it may be replaced by an alternative model should that be available.

For illustration, Fig. 14 presents a sample of the displacement time-histories at $U=60 \mathrm{~m} / \mathrm{s}$ (cf. Fig. 14, top) and the RMS of the displacements for the selected models (cf. Fig. 14, bottom). Based on the RMS, the
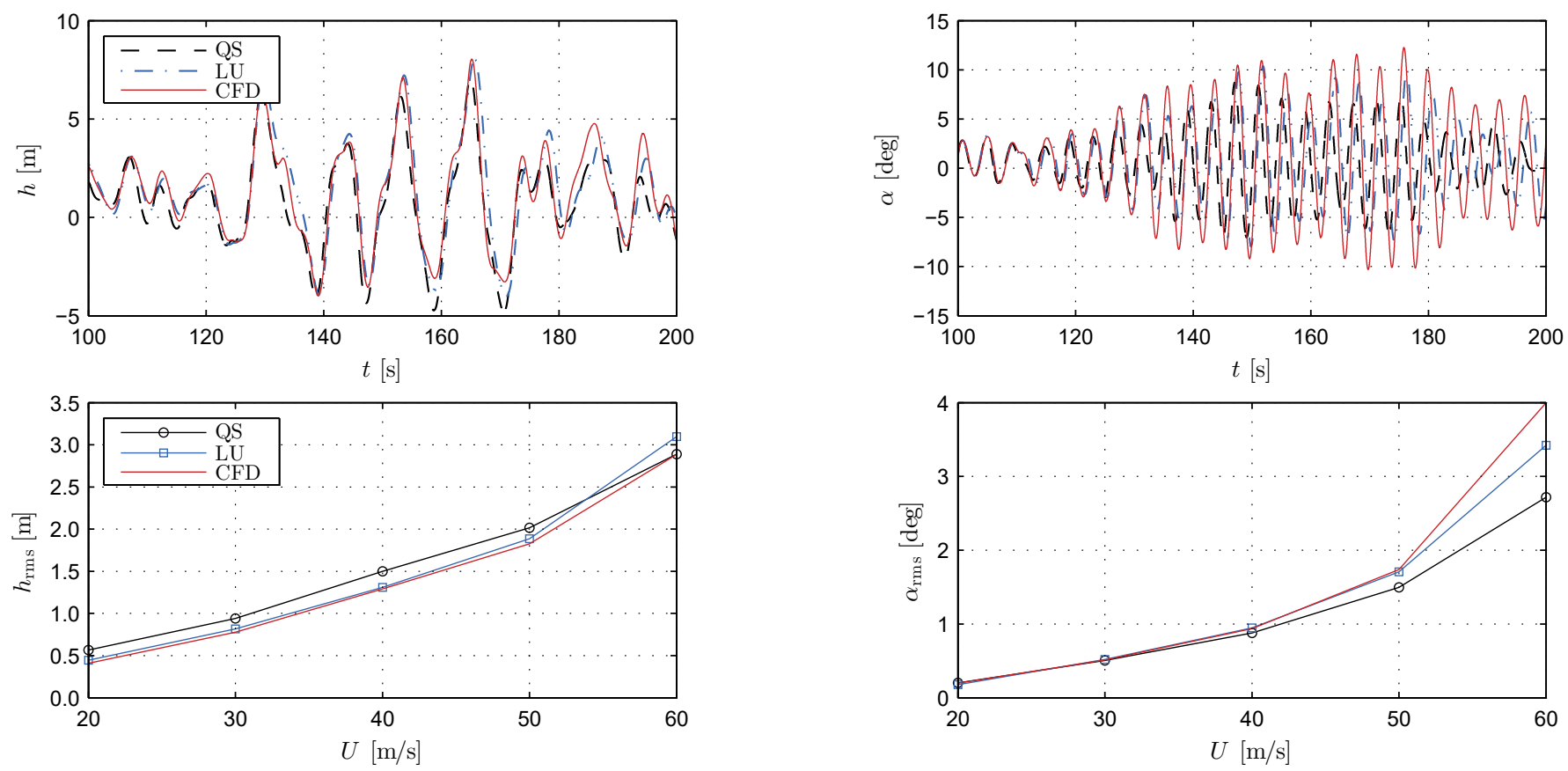

Fig. 14. Sample time-histories at $U=60 \mathrm{~m} / \mathrm{s}$ (top) and RMS (bottom) of the vertical displacements $h$ (left) and rotation $\alpha$ (right). 

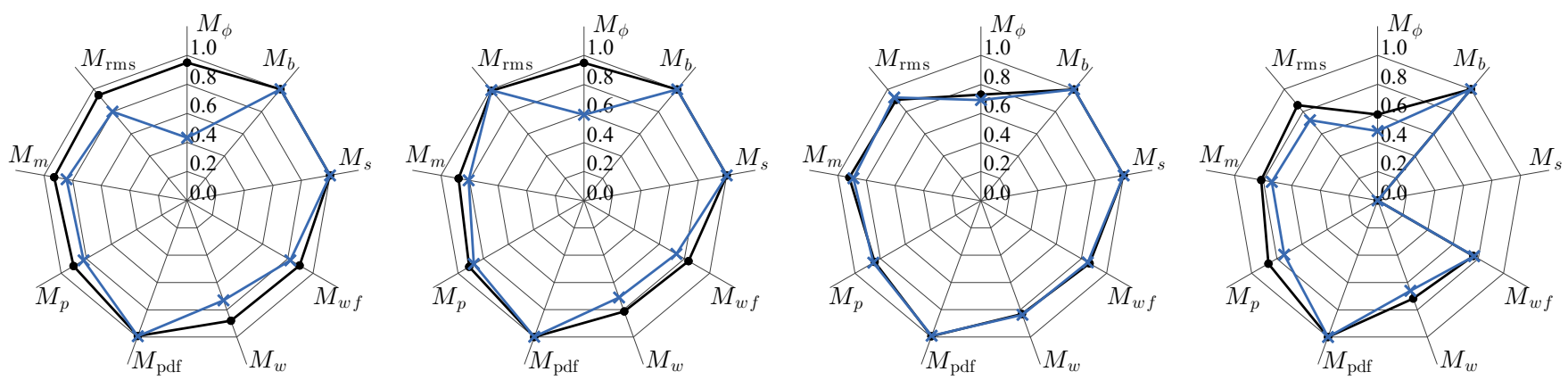

Fig. 15. Comparison metrics for the aeroelastic displacements from buffeting analysis for the LU (black, circle) and QS (blue, cross) models with respect to the CFD model. From left to right: vertical displacements $h(U=30 \mathrm{~m} / \mathrm{s})$, rotation $\alpha(U=30 \mathrm{~m} / \mathrm{s})$, vertical displacements $h(U=60 \mathrm{~m} / \mathrm{s})$, rotation $\alpha(U=60 \mathrm{~m} / \mathrm{s})$.

LU model performs better than the QS model for the vertical displacements, except at $U=60 \mathrm{~m} / \mathrm{s}$. For the torsional displacements, the LU model has smaller discrepancies with respect to the reference CFD model than the QS model, especially at high wind speeds.

To further study the effect of linear fluid memory and quasi-steady nonlinearity, the comparison metrics are computed for two representative wind speeds of $U=30 \mathrm{~m} / \mathrm{s}$ and $U=60 \mathrm{~m} / \mathrm{s}$ (cf. Fig 15). The time-histories considered are with a duration of $T=600 \mathrm{~s}$, re-sampled at sampling frequency $f_{s}=30 \mathrm{~Hz}$. Corresponding to the metric parameters for the generic signals, the following are modified: normalization time $T_{c}=1 /\left(4 f_{h}+4 f_{\alpha}\right)$, and wavelet central frequency $f_{0}=2 \mathrm{~Hz}$.

Generally, the quasi-steady assumption is more rigorous than the linearity, as the comparison metrics for the LU models attain higher values than for the QS model. Looking at the phase metric $M_{\phi}$, the quasi-steady assumption influences the phase significantly, although a phase-difference is apparent for the high wind speed for the LU model. In case of the rotation at $U=30 \mathrm{~m} / \mathrm{s}$, it can be observed that while RMS metrics $M_{\mathrm{rms}}^{\alpha_{\mathrm{CFD}}, \alpha_{\mathrm{LU}}} \approx M_{\mathrm{rms}}^{\alpha_{\mathrm{CFD}}, \alpha_{\mathrm{QS}}} \approx 1$, the magnitude metrics $M_{m}^{\alpha_{\mathrm{CFD}}, \alpha_{\mathrm{LU}}}$ and $M_{m}^{\alpha_{\mathrm{CFD}}, \alpha_{\mathrm{QS}}}$ are lower than 1 (cf. Fig 15, center-left). This indicates that the localized time behavior of the signals is not as similar as for the global and averaged quantities. Moreover, by studying the comparison metrics for the vertical displacements $h$ at $U=60$ $\mathrm{m} / \mathrm{s}$ (cf. Fig 15, center-right), it can realized why considering the RMS as an only basis for comparison is insufficient. Particularly, the QS model performs slightly better for the RMS metric $M_{\mathrm{rms}}^{h_{\mathrm{CFD}}, h_{\mathrm{LU}}}<M_{\mathrm{rms}}^{h_{\mathrm{CPD}}, h_{\mathrm{QS}}}$, while the situation is reverse for the magnitude metric, i.e. $M_{\mathrm{m}}^{h_{\mathrm{CFD}}, h_{\mathrm{LU}}}>M_{\mathrm{m}}^{h_{\mathrm{CFD}}, h_{\mathrm{QS}}}$. This indicates that only the global quantities of the CFD model are in better correspondence with the QS model and not the local ones. Since the input may be considered as identical, to draw a general conclusion that the quasi-steady nonlinearity is more critical than the linear fluid memory at high wind speeds, all metrics should support such statement. In this case, it may only indicated that the influence of the nonlinearity becomes apparent in the RMS at high wind speeds.

The PDF metrics resulted in values $M_{\mathrm{pdf}} \approx 1$, mainly because there is general agreement between the higher-order moments of the normalized PDFs, with discrepancies at the tail. These extreme values are covered in the peak metric. In case the tail of the PDF is of interest, another statistical divergence measure should be utilized instead of the Bhattacharyya distance.

Except for the rotation at $U=60 \mathrm{~m} / \mathrm{s}$, the wavelet metric for the LU model is similar to the frequencynormalized wavelet metric, i.e. $M_{w}^{\mathrm{CFD}, \mathrm{LU}} \approx M_{w f}^{\mathrm{CFD}, \mathrm{LU}}$. Hence, the discrepancy in the wavelet metric is due to the magnitude difference, while the relative frequency content is the same. This can be observed in the wavelet magnitude for the vertical displacements at $U=60 \mathrm{~m} / \mathrm{s}$ (cf. Fig. 16, top). Moreover, a spectral contribution at a frequency corresponding to $f_{\alpha}=0.287 \mathrm{~Hz}$ can also be observed for the wavelet magnitude 

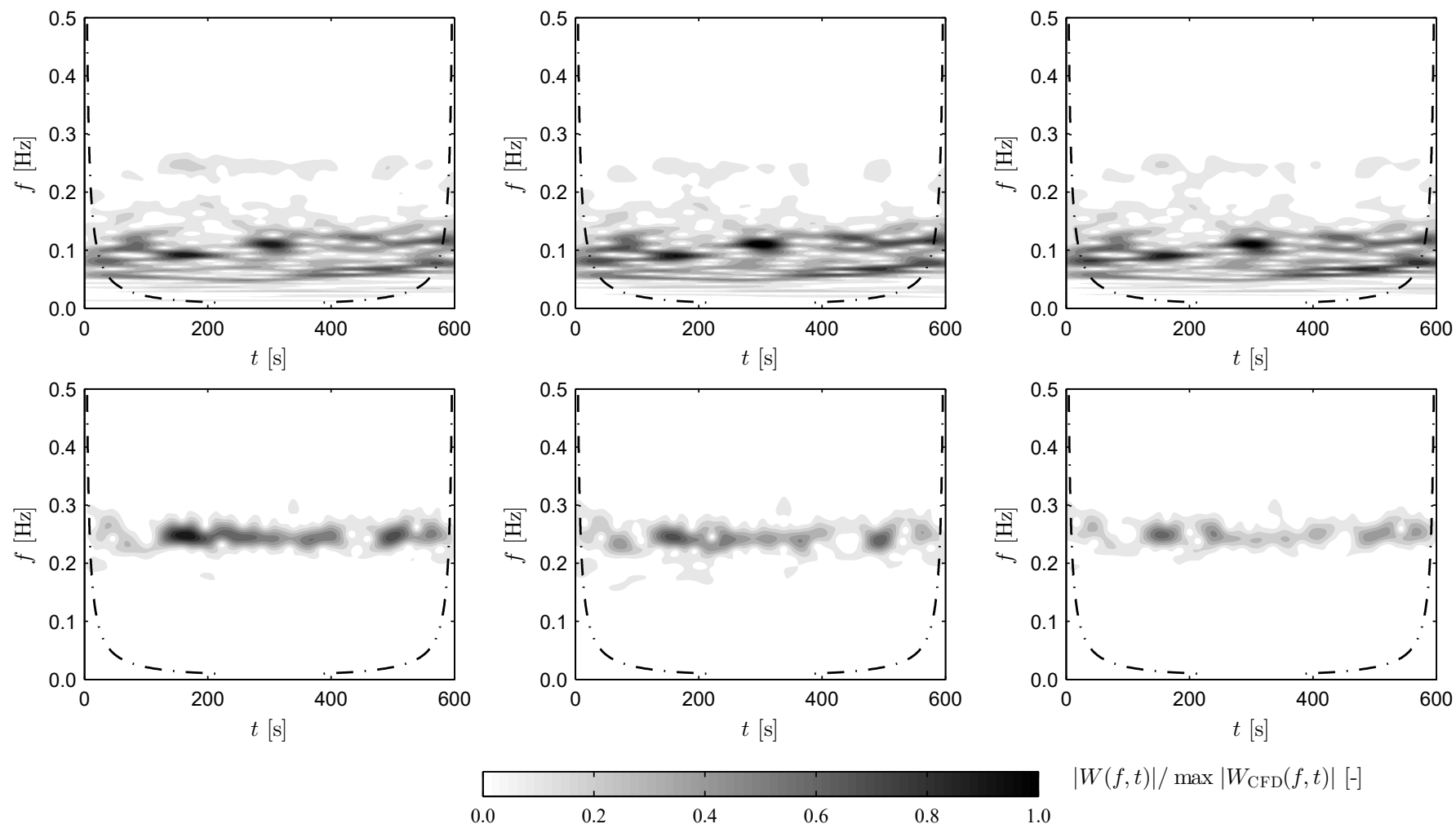

$|W(f, t)| / \max \left|W_{\mathrm{CFD}}(f, t)\right|[-]$

Fig. 16. Wavelet amplitude $|W(f, t)|$ of the vertical displacements $h$ (top) and rotation $\alpha$ (bottom) for the CFD (left), LU (center) and QS (right) models from buffeting analysis at $U=60 \mathrm{~m} / \mathrm{s}$. The dashed-dot line indicates the cone of influence.

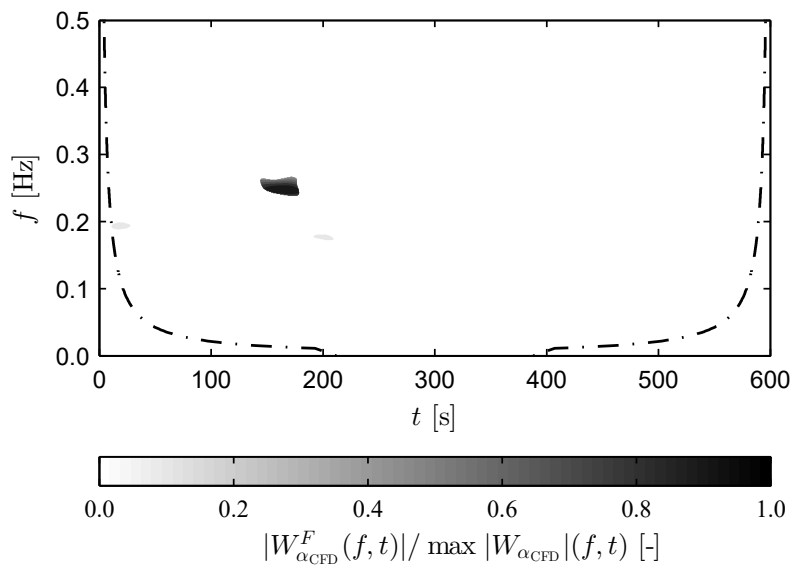

Fig. 17. Filtered nonstationary part of the wavelet amplitude for the rotation $\left|W_{\alpha_{\mathrm{CFD}}}^{F}\right|$ at $U=60 \mathrm{~m} / \mathrm{s}$. The dashed-dot line indicates the cone of influence.

of the vertical displacements $h$ (cf. Fig. 16, top), indicating that the aerodynamic coupling is significant for all models. Overall, the wavelet-based metrics indicated that the LU model performs better than the QS model. Further, not having a similar value for the wavelet-based metrics for the QS model $M_{w}^{h_{\mathrm{CFD}}, h_{\mathrm{QS}}} \approx M_{w f}^{h_{\mathrm{CFD}}, h_{\mathrm{QS}}}$ at $U=30 \mathrm{~m} / \mathrm{s}$ indicates that the fluid memory influences the relative frequency content (cf. Fig 15, left). 
Predominantly, the comparison metrics for both models indicated largest discrepancies for the rotation $\alpha$ at $U=60 \mathrm{~m} / \mathrm{s}$ (cf. Fig 15, right). Hence, both models are unable to completely replicate the aerodynamic moment at high wind speeds, as noted in Kavrakov and Morgenthal (2018b). Having $M_{w} \leq 0.8$ and $M_{w} \approx M_{w f}$ for both models indicates that there is a difference in both magnitude and relative frequency content. From the wavelet magnitude (cf. Fig. 16, bottom), high amplitudes can be observed for the rotation $\alpha$ for the CFD model in the approximate time interval $100 \mathrm{~s} \lesssim t \lesssim 200 \mathrm{~s}$, which are not well captured by the semi-analytical models. This is also depicted in the sample time-history of the rotation (cf. Fig. 14, top-right). Moreover, this particular portion of the time-history is detected to be nonstationary for the CFD model, as shown in Fig. 17. For a stationary input, which is the case for the wind fluctuations in this study, only a nonlinear model may result in a nonstationary output in the stable range (i.e. not in the flutter region). Hence, the unsteady nonlinearity in the self-excited forces seems like a plausible reason for the amplitude modulations in this interval (Kavrakov and Morgenthal 2018b). Since the response for the both semi-analytical models is detected to be stationary, the stationarity metric yields $M_{s}^{\alpha_{\mathrm{CFD}}, \alpha_{\mathrm{LU}}}=M_{s}^{\alpha_{\mathrm{CFD}}, \alpha_{\mathrm{QS}}}=0$.

The bispectrum showed no appreciable peaks for all models; thus, the bispectrum metric amounts to $M_{b}=1$. Although quadratic phase coupling may be occurring, this effect can be further reduced by the mechanical admittance when looking at the dynamic response. Hence, it is easier to be captured for the self-excited aerodynamic forces due to sinusoidal forced excitation, as shown in the following section. It should also be noted that quadratic phase coupling is just one particular type of nonlinearity. Other types of nonlinearities such as higher-order amplitude dependence of the main harmonic (Zhang et al. 2017) in terms of, for e.g., cubic phase coupling cannot be identified by the bispectrum.

\section{Self-excited forces}

The effect of aerodynamic nonlinearity can be prominent in the self-excited forces at large angles of attack. In the following, the self-excited forces due to a sinusoidal forced excitation are compared for the CFD and LU models utilizing the proposed comparison metrics. The quantity of interest, in this case, is the time-history of the self-excited fluctuating moment coefficient due to rotation $C_{M \alpha}^{*}$. Figure 18 (left) depicts a portion of the time-history of $C_{M \alpha}^{*}$, due to rotation about a static angle of attack amounting to $\alpha_{s}=4$ deg for both CFD and LU models. The deck is forced to rotate in a sinusoidal manner with an amplitude of $\alpha_{0}=3 \mathrm{deg}$ and a frequency of $f_{\alpha}=0.041 \mathrm{~Hz}$, which corresponds to a reduced velocity amounting to $V_{r}=U /\left(f_{\alpha} B\right)=16$. As observed from the time-histories, the linear fit for a single harmonic frequency by
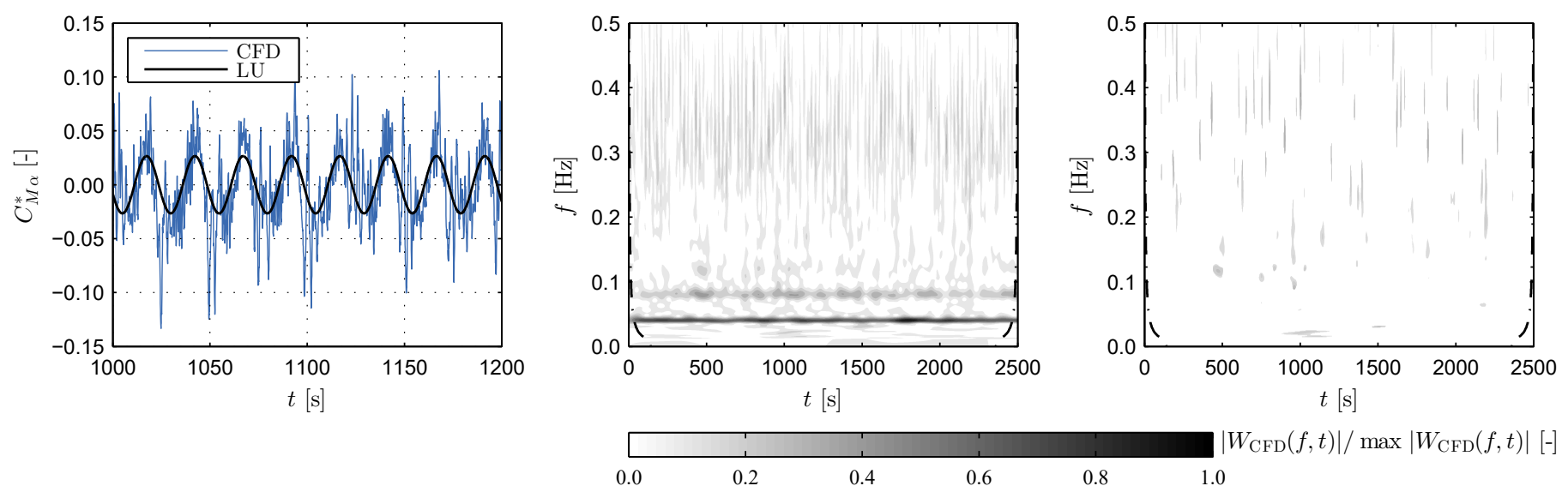

Fig. 18. Sample time-history of the fluctuating moment coefficient $C_{M \alpha}^{*}$ (left) for sinusoidal excitation. Amplitude $\left|W_{C_{M \alpha}^{*}}\right|$ (center) and filtered amplitude $\left|W_{C_{M \alpha}^{*}}^{F}\right|$ (right) of the wavelet coefficients for the CFD model. The dashed-dot line indicates the cone of influence. 


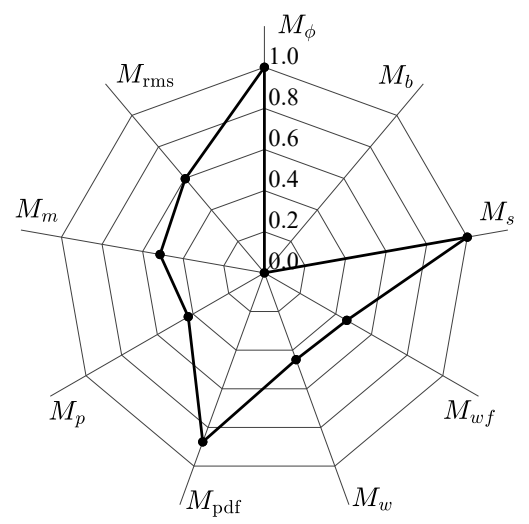

Fig. 19. Comparison metrics for the fluctuating moment coefficient $M_{\mathrm{M} \alpha, \mathrm{CFD}}^{C^{*}, C_{M \alpha, \mathrm{LU}}^{*}}$ for sinusoidal excitation.

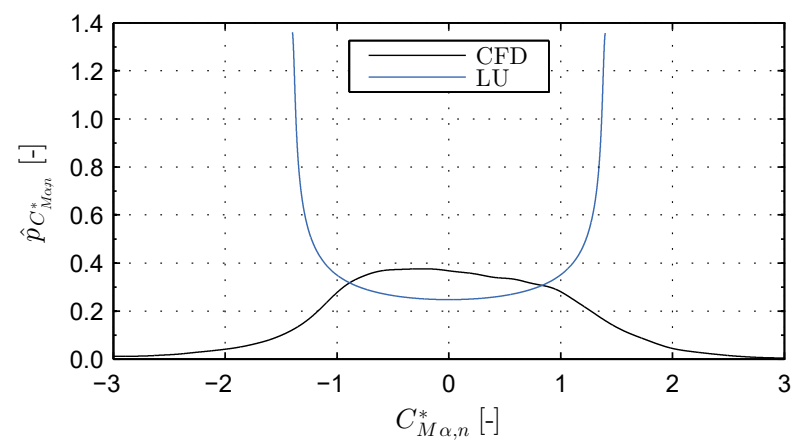

Fig. 20. Estimated PDF of the normalized (standard score) fluctuating moment coefficient $\hat{p}_{C_{M \alpha, n}^{*}}$ for sinusoidal excitation.

the LU model is insufficient to completely describe the complex behavior for the CFD model at this angle of attack (Kavrakov and Morgenthal 2018b).

To quantify the discrepancies between the two time-histories, the comparison metrics are given in Fig. 19 taking the CFD model as a reference again. For the computation of the comparison metrics, the signals considered are with a duration of $T=2500 \mathrm{~s}$ and a sampling frequency amounting to $f_{s}=20 \mathrm{~Hz}$. Modified metric parameters with respect to the ones for the generic signals are the wavelet central frequency, taken as $f_{0}=2 \mathrm{~Hz}$, and the normalization time amounting to $T_{c}=1 /\left(2 f_{\alpha}\right)$ for the phase metric. Both the wavelet

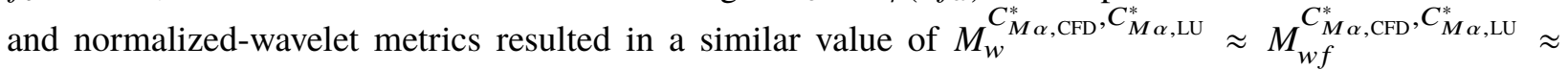
0.45 meaning that there is significant discrepancy in the time-frequency plane mainly due to frequency modulations. This is mostly a consequence of the additional superharmonic contribution in $C_{M \alpha}^{*}$ for the CFD model at $f=2 f_{\alpha}=0.082 \mathrm{~Hz}$, which can be observed in the wavelet coefficients (cf. Fig. 18, center). Moreover, there is a widespread frequency content in the range between $0.2 \mathrm{~Hz}$ and $0.4 \mathrm{~Hz}$, which corresponds to the frequency range of vortex-shedding. Since the LU model is linear, it models only a single harmonic component; hence, it does not account for the frequency content other than the forcing frequency. In terms of signal energy and magnitude, the LU model underestimates the response and the metrics corresponding to these quantities amount to $M_{\mathrm{rms}}^{C_{M \alpha, \mathrm{CFD}}^{*}, C_{M \alpha, \mathrm{LU}}^{*}} \approx 0.6$ and $M_{m}^{C_{M \alpha, \mathrm{CFD}}^{*}, C_{M \alpha, \mathrm{LU}}^{*}} \approx 0.5$, respectively.

Figure 20 depicts the estimated PDFs of the CFD and LU model. The estimated PDF of the CFD model is somewhat Gaussian due to the vortex shedding and interior noise, despite single-frequency input. Since 


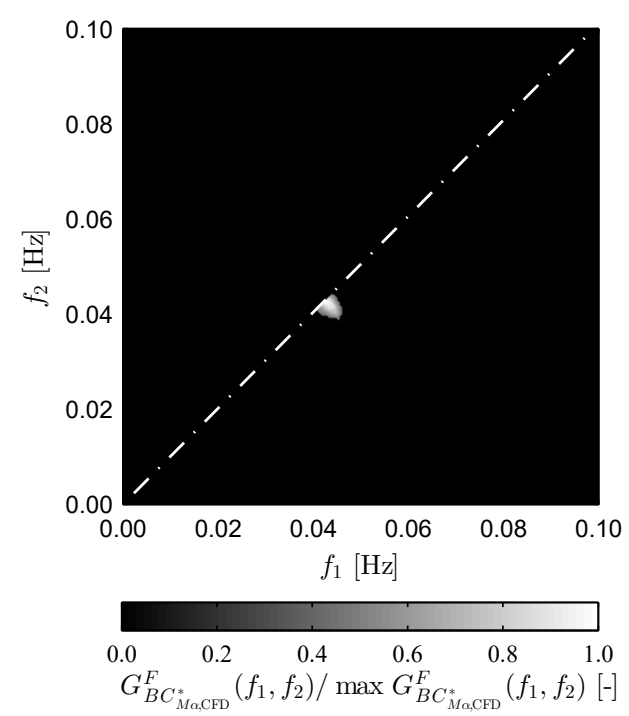

Fig. 21. Amplitude of the filtered phase-randomized bispectrum of the fluctuating moment coefficient $G_{B C_{M \alpha, \mathrm{CFD}}^{*}}^{F}\left(f_{1}, f_{2}\right)$ for sinusoidal excitation. The dashed-dot line indicates the inner triangle.

the LU model is a pure sinusoid, the PDF attains vertical asymptote corresponding to the amplitude of the sinusoid. The PDF metric results to $M_{\mathrm{pdf}}^{C_{\mathrm{M} \alpha, \mathrm{CFD}}^{*}, C_{M \alpha, \mathrm{LU}}^{*}}=0.86$.

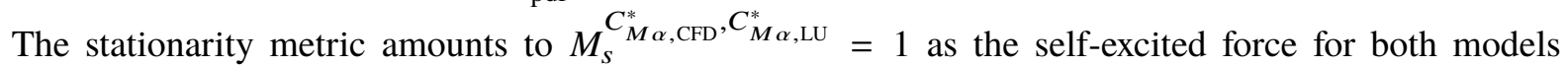
is identified as stationary according to the global criterion (cf. Eq. (16)). The local criterion identified nonstationary part in the wavelet coefficients of the self-excited force for the CFD model. This filtered nonstationary part is given in Fig. 18 (right). Most of the nonstationary contribution is concentrated as sharp peaks at the high frequencies, yielding the premise that this part is due to vortex shedding and local turbulence effects. However, it is argued that this contribution is due to spurious peaks that may appear for the local method for identifying stationarity, as discussed previously for the generic signals. Therefore, both signals are considered to be stationary as identified by the two-level procedure.

As the bispectrum metric resulted in a value $M_{b}^{C_{M \alpha, \mathrm{CFD}}^{*}, C_{M \alpha, \mathrm{LU}}^{*}}=0$, it can be concluded that the additional frequency component at $f=2 f_{\alpha}=0.082 \mathrm{~Hz}$ for the CFD model is due to quadratic phase coupling, i.e. quadratic nonlinearity. This can be also observed from the filtered bispectrum of $C_{M \alpha}^{*}$ for the CFD model, which is depicted in Fig. 21. A distinctively high value is present at the frequency pair $\left(f_{1}, f_{2}\right)=\left(f_{\alpha}, f_{\alpha}\right)$. Further, contribution of the superharmonic component at $2 f_{\alpha}$ is stationary and has relatively higher magnitude than the vortex shedding and local nonstationary effects (cf. Fig. 18, center).

\section{SUMMARY AND CONCLUSIONS}

In summary, this paper used a set of comparison metrics to quantify the discrepancies of particular features between two time-histories in the field of bridge aerodynamics. Nine metrics were considered including seven adapted from previous studies based on the phase, peak, RMS, magnitude, PDF, wavelet, frequency-normalized wavelet and recast for the present application. Two additional metrics were introduced in this study, based on the stationarity and nonlinearity using bispectrum. All of the metrics were constructed in a unified manner, yielding a multi-criteria assessment framework, tailored for comparative studies in bridge aerodynamics.

Initially, the performance of the comparison metrics was examined using controlled numerical experiments by employing generic signals. By modifying particular signal features, the efficacy of the comparison 
metrics was examined. This demonstrated that various global and local signal features should be considered when comparing two time-histories.

Finally, the comparison metrics were applied to a practical example from bridge aerodynamics in terms of the buffeting response and self-excited forces. For the first case, the influence of the quasi-steady and linear unsteady assumptions in buffeting analysis was examined. It was demonstrated that comparing two time-histories based only on the RMS, as per common practice, might yield incomplete conclusions for nonlinear models. The introduced stationarity metric identified the portion of the response with the largest discrepancies as nonstationary for the CFD model, inferring that the response in this region is nonlinear. Such a feature cannot be clearly identified by the rest of the metrics, making this metric important for comparison of nonlinear models. Thus, a multi-criteria assessment of the time-dependent response is pertinent to study meticulously the influence of aerodynamic assumptions. This was further echoed in the comparison of the self-excited forces due to forced rotation. In this case, the proposed bispectrum metric was able to identify the inability of the LU model to replicate the aerodynamic nonlinearity in terms of quadratic phase coupling. This revealed that the discrepancies in the other metrics are not simply due to vortex-shedding or numerical noise, but rather due to the nature of a particular nonlinear feature.

In conclusion, the utilization of a set of comparison metrics offers a deeper insight into the comparison between aerodynamic models for aeroelastic analyses of bridges. By employing the comparison metrics, discrepancies in particular signal features for two models can be quantified separately. The outcome of this study is meant to offer an alternative perspective to model comparison and validation, in order to facilitate a well-informed model evaluation in the field of bridge aerodynamics and other areas involving dynamic systems with potential nonstationarity, nonlinearity and memory effects. Presently, all metrics are considered individually as some of them may be redundant. Future studies may involve combining the metrics in a unified metric by utilizing weighting factors.

\section{DATA AVAILABILITY STATEMENT}

Some or all data, models, or code generated or used during the study are available in a repository online in accordance with funder data retention policies. The matlab code for the comparison metrics, including partial results, is available online https://github.com/IgorKavrakov/CompMet. Some data, models, or code generated or used during the study are proprietary or confidential in nature and may only be provided with restrictions.

\section{ACKNOWLEDGEMENTS}

IK and GM gratefully acknowledge the support by the German Research Foundation (DFG) [Projects No. 43475018 and 329120866] throughout IK's studies and research stay at the Department of Civil and Environmental Engineering and Earth Sciences at the University of Notre Dame, USA. AK acknowledges the support provided in part by the NSF Grant \#1562244.

\section{REFERENCES}

Abbas, T., Kavrakov, I., and Morgenthal, G. (2017). "Methods for flutter stability analysis of long-span bridges: A review." Proc. Inst. Civ. Eng. - Bridge Eng., 170, 271-310.

Akbari, M. H. and Price, S. J. (2003). "Simulation of dynamic stall for a NACA 0012 airfoil using a vortex method." J. Fluids Struct., 17, 855-874.

Bliemel, F. (1973). “Theil's forecast accuracy coefficient:A clarification.” J. Mark. Res., 10, 444-446.

Borgant, P., Flandrin, P., Hoene, P., Richard, C., and Xiao, J. (2010). "Testing stationarity with surrogates:A time-frequency approach.” IEEE Trans. Signal Process., 58, 3459-3470.

Botev, Z., Grotowski, J., and Kroese, D. (2010). "Kernel density estimation via diffusion." Ann. Stat., 38, 2916-2957.

Caracoglia, L. and Jones, N. P. (2003). "Time domain vs frequency domain characterization of aeroelastic forces for bridge deck sections." J. Wind. Eng. Ind. Aerodyn., 91, 371-402. 
Carassale, L., Wu, T., and Kareem, A. (2014). "Nonlinear aerodynamics and aerielastic analysis of bridges: Frequency domain approach.” J. Eng. Mech., 140(8), 04014051.

Chen, X. and Kareem, A. (2001). "Aeroelastic analysis of bridges under multicorrelated winds: Integrated state-space approach.” J. Eng. Mech., 127(11), 1124-1134.

Chen, X. and Kareem, A. (2002). "Advances in modeling of aerodynamic forces on bridge decks." J. Eng. Mech., 128(11), 1193-1205.

Chen, X. and Kareem, A. (2003). "Aeroelastic analysis of bridges: Effects of turbulence and aerodynamic nonlinearities." J. Eng. Mech., 129(8), 885-895.

Chen, X. and Kareem, A. (2006). "Revisiting multimode coupled bridge flutter: Some new insights." J. Eng. Mech., 132(10), 1115-1123.

Cottet, G. and Koumoutsakos, P. (2000). Vortex methods: Theory and Practice. Cambridge University Press.

Cvetkovic, D., Übely, E. D., and Cosic, I. (2008). "Wavelet transform feature extraction from human PPG, ECG, and EEG signal responses to ELF PEMF exposures: A pilot study." Dig. Signal Process., 5, 861-874.

Diana, G., Rocchi, D., and Argentini, T. (2013). "An experimental validation of band superposition model of the aerodynamic forces acting on a multi-box deck sections." J. Wind Eng. Ind. Aerodyn., 113, 40-58.

Diana, G., Rocchi, D., Argentini, T., and Muggiasca, S. (2010). "Aerodynamic instability of a bridge deck section model: Linear and nonlinear approach to force modeling." J. Wind Eng. Ind. Aerodyn., 98, 363-374.

Diana, G., Stoyanoff, S., Aas-Jakobsen, K., Allsop, A., Andersen, M., Argentini, T., Montoya, M. C., , Hernández, S., Juardo, J. A., Katsuchi, H., Kavrakov, I., Kim, H., Larose, G., Larsen, A., Morgenthal, G., Øiseth, O., Omarini, S., Rocchi, D., Svendsen, M., and Wu, T. (2019a). "IABSE Task Group 3.1 benchmark results. Part 1: Numerical analysis of a two-degree-of- freedom bridge deck section based on analytical aerodynamics." Struct. Eng. Int. doi:10.1080/10168664.2019.1639480.

Diana, G., Stoyanoff, S., Aas-Jakobsen, K., Allsop, A., Andersen, M., Argentini, T., Montoya, M. C., , Hernández, S., Juardo, J. A., Katsuchi, H., Kavrakov, I., Kim, H., Larose, G., Larsen, A., Morgenthal, G., Øiseth, O., Omarini, S., Rocchi, D., Svendsen, M., and Wu, T. (2019b). "IABSE Task Group 3.1 benchmark results. Part 2: Numerical analysis of a 3-degree-of-freedom bridge deck section based on experimental aerodynamics.” Struct. Eng. Int. doi:10.1080/10168664.2019.1661331.

Eldrege, J. D. (2007). "Numerical simulation of the fluid dynamics of 2D rigid body motion with the vortex particle method." J. Comput. Phys., 221, 626-648.

Elgar, S. and Sebert, G. (1989). "Statistics of bicoherence and biphase." J. Geophys. Res., 94, 993-998.

Fackrell, J. W. A. and McLaughlin, S. (1995). "Quadratic phase coupling detection using higher order statistics." IEE Colloquium on Higher Order Statistics in Signal Processing: Are They of Any Use?, 9/1-9/8.

Fackrell, J. W. A., White, P. R., Hammond, J. K., and Pinnington, R. J. (1995). "The interpretation of the bispectra of vibration signals-I. Theory." Mech. Syst. Signal Process., 9, 257-266.

Fung, Y. (1993). An introduction to the theory of aeroelasticity. Dover Publications, New York, dover edition.

Ge, Y. and Xiang, H. F. (2008). "Computational models and methods for aerodynamic flutter of long-span bridges.” J. Wind Eng. Ind. Aerodyn., 96, 1912-1924.

Grossmann, A. and Morlet, J. (1984). "Decomposition of hardy functions into square integrable wavelets of constant shape.” SIAM J. Math. Anal., 15, 723-736.

Gurley, K. and Kareem, A. (1997). "Analysis interpretation modeling and simulation of unsteady wind and pressure data." J. Wind Eng. Ind. Aerodyn., 69-71, 657-669.

Gurley, K., Kijewski, T., and Kareem, A. (2003). "First- and higher-order correlation detection using wavelet transforms." J. Eng. Mech., 129, 188-201.

Hora, J. and Campos, P. (2015). "A review of performance crietria to validate simulation models." Expert Syst., 32, 578-595.

Huang, L., Xu, Y.-L., and Liao, H. (2014). "Nonlinear aerodynamic forces on thin flat plate: Numerical 
study." J. Fluids. Struct., 44, 182-194.

Jamšek, J., Stefanovska, A., and McClintock, P. V. E. (2007). "Wavelet bispectral analysis for the study of interactions among oscillators whose basic frequencies are significantly time variable." Phys. Rev. E, 76, 046221-046221-11.

Jiang, X. and Mahadevan, S. (2010). "Wavelet spectrum analysis approach to model validation of dynamic systems.” Mech. Syst. Signal Process., 25, 575-590.

Kareem, A. and Kijewski (2002). "Time-frequency analysis of wind effects on structures." J. Wind Eng. Ind. Aerody., 90, 1435-1452.

Kareem, A. and Wu, T. (2013). "Wind-induced effects on bluff bodies in turbulent flows:Nonstationary, non-Gaussian and nonlienar features." J. Wind Eng. Ind. Aerody., 122, 21-37.

Kavrakov, I., Legatiuk, D., Gürlebeck, K., and Morgenthal, G. (2019). "A categorical perspective towards aerodynamic models for aeroelastic analyses of bridge decks." $R$. Soc. Open Sci., 6, 181848.

Kavrakov, I. and Morgenthal, G. (2017). "A comparative assesment of aerodynamic models for buffeting and flutter of long-span bridges." Eng., 3, 823-838.

Kavrakov, I. and Morgenthal, G. (2018a). "Aeroelastic analyses of bridges using a Pseudo-3D vortex method and velocity-based synthetic turbulence generation.” Eng. Struct., 176, 825-839.

Kavrakov, I. and Morgenthal, G. (2018b). "A synergistic study of a CFD and semi-analytical models for aeroelastic analysis of bridges in turbulent wind conditions." J. Fluids Struct., 82, 59-85.

Kijewski, T. and Kareem, A. (2003). "Wavelet transforms for system identification in civil engineering." Comput.-Aided Civ. Infrastrcut. Eng., 18, 339-355.

Kim, T., Powers, E. J., Grady, W. M., and Arapostathis, A. (2007). "A novel qpc detector for the health monitoring of rotating machines." IEEE Instrumentation Measurement Technology Conference, 1-6.

Kim, Y. C. and Powers, E. J. (1979). "Digital bispectral analysis and its applications to nonlinear wave interactions." IEEE Trans. Plasma Sci., 7, 120-131.

Kovacs, I., Svensson, H. S., and Jordet, E. (1992). "Analytical aerodynamic investigation of cable-stayed Helgeland bridge.” J. Struct. Eng., 118, 147-168.

Kristeková, M., Kristek, J., and Moczo, P. (2009). "Time-frequency misfit and goodness-of-fit criteria for quantitative comparison of time signals." Geophys. J. Int., 178, 813-825.

Lancester, G., Iatsenko, D., Pidde, A., Ticcinelli, V., and Stefanovska, A. (2018). "Surrogate data for hypothesis testing of physical systems." Phys. Rep., 748, 1-60.

Larsen, A. and Walther, J. H. (1997). "Aeroelastic analysis of bridge girder sections based on discrete vortex simulations." J. Wind Eng. Ind. Aerodyn., 67\&68, 253-265.

Li, X., Duan, L., Logan, J. V., and Sleigh, J. W. (2009). "The comodulation measure of neuronal oscillations with general harmonic wavelet bicoherence and application to sleep analysis." NeuroImage, 48, 501-514.

Li, Y. and Kareem, A. (1990). "Stochastic response of tension leg platforms to wind and wave fields." $J$. Wind Eng. Ind. Aerodyn., 36, 915-926.

McCullough, M. (2016). "Data-driven models: From data to knowledge for extreme events." Ph.D. thesis, University of Notre Dame, University of Notre Dame.

McCullough, M. and Kareem, A. (2013). "Testing stationarity with wavelet-based surrogates." J. Eng. Mech., 139, 200-209.

Morgenthal, G., Corriols, A., and Bendig, B. (2014). "A GPU-accelerated Pseudo-3D vortex method for aerodynamic analysis." J. Wind Eng. Ind. Aerodyn., 125, 69-80.

Morgenthal, G. and McRobie, F. (2002). "A comparative study of numerical methods for fluid-structure interaction analysis in long-span bridge design." Wind Struct., 5, 101-114.

Morgenthal, G. and Walther, J. (2007). "An immersed interface method for the Vortex-In-Cell algorithm." Comput. Struct., 85, 712-726.

Müller, M. (2007). Information retrieval for music and motion. Springer.

Nielsen, F. and Boltz, S. (2011). "The Burbea-Rao and Bhattacharyya centroids." IEEE Trans. Inf. Theory, 
$57,5455-5466$.

Nikitas, C. and Petropulu, A. P. (1993). Higher-Order Spectra Analysis: A Non-Linear Signal Processing Framework. Prentice Hall.

Øiseth, O., Rönnquist, A., and Sigbjörnsson (2011). "Time domain modeling of self-excited aerodynamic forces for cable supported bridges: A comparative study." Comput. Struct., 89, 1306-1322.

Pardo, L. (2006). Statistical Inference Based on Divergence Measures. Chapman \& Hall/CRC, Taylor \& Francis Group.

Petrini, F., Giuliano, F., and Bontempi, F. (2007). "Comparison of time domain techniques for evaluation of the response of the stability in long span suspension bridges." Comput. Struct., 85, 1032-1048.

Richard, C., Ferrari, A., Amoud, H., Honeine, P., Flandrin, P., and Borgnat, P. (2010). "Statistical hypothesis testing with time-frequency surrogates to check signal stationarity." IEEE International Conference on Acoustics, Speech and Signal Processing, 3666-3669.

Sarin, H., Kokkolaras, M., Hulbert, G., Papalambros, P., Barbat, S., and Yang, R. J. (2010). "Comparing time histories for validation of simulation models:Error measures and metrics." J. Dyn. Syst. Meas. Contr., 132, 061401-061401-10.

Scanlan, R. H. (1978). "The action of flexible bridges under wind, I: Flutter theory.” J. Sound. Vib., 60(2), 187-199.

Scanlan, R. H. (2001). "Reexamination of sectional aerodynamic force functions for bridges." J. Wind. Eng. Ind. Aerodyn., 89, 1257-1266.

Scanlan, R. H., Bélveau, J. G., and Budlong, K. S. (1974). "Indicial aerodynamic functions for bridge decks." J. Eng. Mech. Div., 100, 657-673.

Scully, C. G., Mitrou, N., Braam, B., Cupples, W. A., and Cho, K. H. (2017). "Detecting interactions between renal autoregulation mechanisms in time and space." IEEE Trans. Biomed. Eng., 64, 690-698.

Shamma, J. S. and Zhao, R. (1993). "Fading-memory feedback systems and robust stability." Automatica, 29, 191-200.

Sprague, M. A. and Geers, T. (2004). "A spectra-element method for modelling cavitation in transient fluid-structure interaction." Int. J. Numer. Meth. Eng., 60, 2467-2499.

Teferra, K., Schields, M. D., Hapij, A., and Daddazio, R. P. (2014). "Mapping model validation metrics to subject matter expert scores for model adequacy assessment." Reliab. Eng. Syst. Safe., 132, 9-19.

van Milligen, B. P., Carreras, B., and Garcia, L. (1995). "Wavelet bicoherence: A new turbulence analysis tool." Phys. Plasmas, 2, 3017-3032.

Wang, L., McCullough, M., and Kareem, A. (2013). "A data-driven approach for simulation of full-scale downburst wind speeds." J. Wind Eng. Ind. Aerodyn., 123, 171-190.

Willmott, C. J., Ackleson, S. G., Davis, R. E., Feddema, J. J., Klink, K. M., Legates, D. R., O’Donnel, J., and Rowe, C. (1985). "Statistics for the evaluation of comparison of models." J. Geophys. Res., 90, 8995-9905.

Wu, T. and Kareem, A. (2011). "Modeling hysteretic nonlinear behavior of bridge aerodynamics via cellular automata nested neural network." J. Wind Eng. Ind. Aerodyn., 99, 378-388.

Wu, T. and Kareem, A. (2013a). "Aerodynamics and aeroelasticity of cable-supported birdges: Identification of nonlinear features." J. Eng. Mech., 139, 1886-1893.

Wu, T. and Kareem, A. (2013b). "Bridge aerodynamics and aeroelasticity: A comparison of modeling schemes." J. Fluids Struct., 43, 347-370.

Wu, T. and Kareem, A. (2013c). "A nonlinear convolution scheme to simulate bridge aerodynamics." Comput. Struct., 128, 259-271.

Wu, T. and Kareem, A. (2015). "A nonlinear analysis framework for bluff-body aerodynamics: A Volterra representation of the solution of Navier-Stokes equations." J. Fluids Struct., 54, 479-502.

Zhang, Z., Zhang, X., Yang, Y., and Ge, Y. (2017). "Nonlinear aerodynamic and energy input properties of a twin-box girder deck section.” J. Fluids Struct., 74, 413-426. 